\title{
Presetting Basal Ganglia for Volitional Actions
}

\author{
Masayuki Watanabe ${ }^{1,2}$ and Douglas P. Munoz ${ }^{1,2,3,4,5}$ \\ ${ }^{1}$ Centre for Neuroscience Studies, ${ }^{2}$ Canadian Institutes of Health Research Group in Sensory-Motor Integration, and Departments of ${ }^{3}$ Physiology, \\ ${ }^{4}$ Psychology, and ${ }^{5}$ Medicine, Queen's University, Kingston, Ontario, Canada K7l 3N6
}

The basal ganglia (BG) have been considered as a key structure for volitional action preparation. Neurons in the striatum, the main BG input stage, increase activity gradually before volitional action initiation. However, because of the diversity of striatal motor commands, such as automatic (sensory driven) and volitional (internally driven) actions, it is still unclear whether an appropriate set of neurons encoding volitional actions are activated selectively for volitional action preparation. Here, using the antisaccade paradigm (look away from a visual stimulus), we dissociated neurons in the caudate nucleus, the oculomotor striatum, encoding predominantly automatic saccades toward the stimulus and volitional saccades in the opposite direction of the stimulus in monkeys. We found that before actual saccade directions were defined by visual stimulus appearance, neurons encoding volitional saccades increased activity with elapsed time from fixation initiation and by a temporal gap between fixation point disappearance and stimulus appearance. Their activity was further enhanced by an antisaccade instruction and correlated with antisaccade behavior. Neurons encoding automatic saccades also increased activity with elapsed time from fixation initiation and by a fixation gap. However, the activity of this type of neuron was not enhanced by an antisaccade instruction nor correlated with antisaccade behavior. We conclude that caudate neurons integrate nonspatial signals, such as elapsed time from fixation initiation, fixation gap, and task instructions, to preset BG circuits in favor of volitional actions to compete against automatic actions even before automatic and volitional commands are programmed with spatial information.

\section{Introduction}

Volitional actions are preceded by preparatory processes (Haggard, 2008). Such preparatory processes are reflected in a gradual increase in cortical activity (Connolly et al., 2002; Colebatch, 2007). The basal ganglia (BG) have a critical role in regulating cortical preparatory activity by their tight anatomical interconnections with specific cortical areas (Alexander et al., 1986). Indeed, patients with several BG disorders show attenuated preparatory cortical activity accompanied with behavioral deficits (Cunnington et al., 1997; Johnson et al., 2001).

The involvement of the BG in volitional action preparation is also supported by the following basic physiological phenomenon observed in behaving animals. Neurons in the striatum, the major input stage of the BG, increase activity gradually before upcoming task events (Hikosaka et al., 1989b; Apicella et al., 1992; Schultz et al., 1992; Takikawa et al., 2002). In the context of motor control, this buildup activity presumably reflects preparatory set (action readiness) (Itoh et al., 2003; Lee and Assad, 2003). The major assumption of this hypothesis is that signals carried by the buildup activity are associated with developing premotor commands in individual striatal neurons so that appropriate striatal neurons are recruited selectively before required volitional ac-

Received April 6, 2010; revised May 21, 2010; accepted May 27, 2010.

This work was supported by the Canadian Institutes of Health Research Grant MOP-77734. D.P.M. was supported by the Canadian Research Chair Program. We thank A. Lablans and R. Cranham for animal care, F. Paquin, S. Hickman, and M. Lewis for technical assistance, and members in Munoz lab for comments on this manuscript.

Correspondence should be addressed to Douglas P. Munoz, Centre for Neuroscience Studies, Queen's University, Room 234, Botterell Hall, 18 Stuart Street, Kingston, Ontario, Canada, K7L3N6. E-mail: doug_munoz@biomed. queensu.ca.

DOI:10.1523/JNEUROSCI.1738-10.2010

Copyright $\odot 2010$ the authors $\quad 0270-6474 / 10 / 3010144-14 \$ 15.00 / 0$ tions. However, it is still unclear whether this assumption is valid because of the diversity of signals encoded by individual striatal neurons, such as sensory-driven and internally driven actions (Hikosaka et al., 1989a; Kimura et al., 1992; Romo et al., 1992; Schultz and Romo, 1992).

Here, we examine this issue by taking advantage of the following behavioral paradigm in which an appropriate association of information carried by preparatory and premotor activity is critical for correct behavioral outcome. The antisaccade paradigm requires subjects to look away from a visual stimulus (Fig. 1A) (Hallett, 1978). This simple requirement dissociates the following two theoretical saccade commands into opposite directions: an automatic (sensory driven) saccade toward the stimulus and a volitional (internally driven) saccade away from the stimulus (Everling and Fischer, 1998; Munoz and Everling, 2004). It has been shown previously that there are different types of neurons in the caudate nucleus, the oculomotor part of the striatum, which encode predominantly either automatic or volitional saccade commands (Watanabe and Munoz, 2009). Correct antisaccade performance requires the appropriate selection of the volitional command over the predominantly automatic command. Accordingly, if the buildup activity in the caudate nucleus is critical for correct antisaccade performance, it should facilitate neurons encoding correct volitional saccade commands selectively, while neurons encoding erroneous automatic saccade commands should be refrained from such facilitation.

To test the above hypothesis, we recorded from single caudate neurons in monkeys performing the antisaccade paradigm. Our results suggest that, before required antisaccade directions were determined by stimulus appearance, the buildup activity of caudate neurons presets BG circuits in favor of volitional saccades to compete against automatic saccades. 
A

\section{Pro- and Antisaccades}

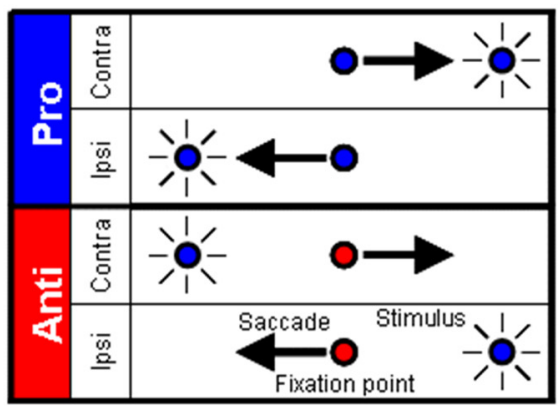

B

\section{Overlap condition}

Fixation point

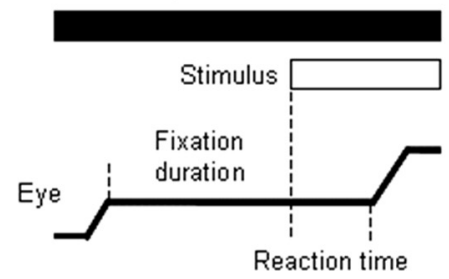

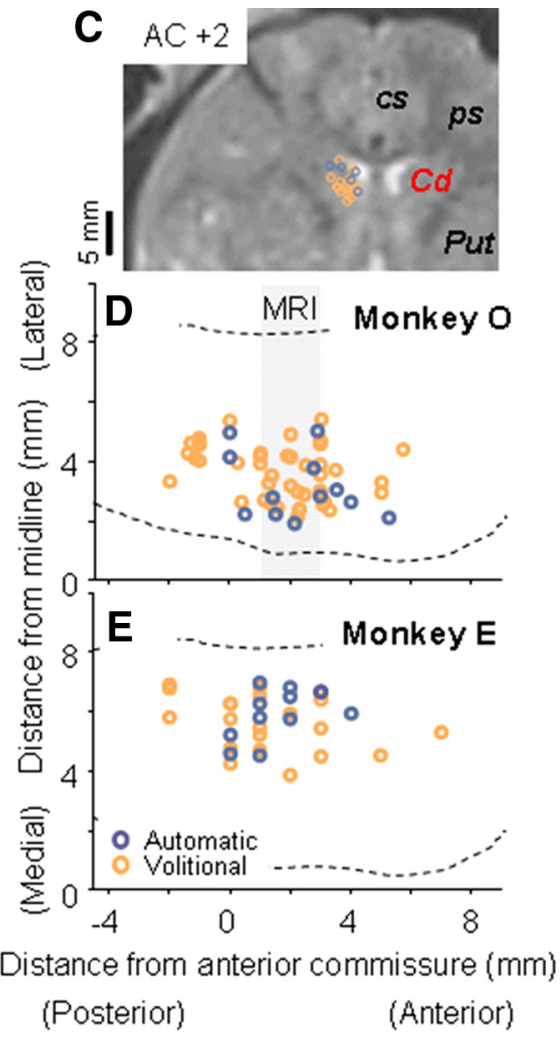

Figure 1. Behavioral paradigm and recording sites. $A$, Prosaccade and antisaccade instructions. Fixation point color indicates monkeys are to perform a prosaccade (look toward a stimulus) or an antisaccade (look away from the stimulus). "Contra" and "Ipsi" indicate saccade directions. $\boldsymbol{B}$, Event time course. After fixation point appearance, monkeys acquired the fixation point and then generated a saccade in response to stimulus appearance. $C, M R$ images $[2 \mathrm{~mm}$ anterior from the anterior commissure $(A C)]$ in monkey 0 with neural recording sites. Cd, Caudate nucleus; Put, putamen; $c$, cingulate sulcus; ps, principal sulcus. $\boldsymbol{D}, \boldsymbol{E}$, Neural recording sites projected on the horizontal plane in monkey $0(\boldsymbol{D})$ and monkey $E(\boldsymbol{E})$, respectively. Sites included in the gray stripes in $\boldsymbol{D}$ are superimposed on the MR image (C). Broken lines indicate the boundaries of the caudate nucleus (Francois et al., 1996). In monkey E, the level of the anterior commissure is estimated at $19 \mathrm{~mm}$ anterior from the intermeatal line (Mikula et al., 2007).

saved their waveforms ( $20 \mathrm{kHz}$ sampling rate), and performed offline sorting analyses. The behavioral paradigm was controlled by a QNXbased real-time data acquisition system (REX, version 5.4) (Hays et al., 1982). The system was then replaced with the Tempo/Win computing system (reflective computing). Visual stimuli (red and green) were back projected on a tangential screen at a distance of $86 \mathrm{~cm}$ from the eye by light-emitting diodes $\left(0.3 \mathrm{~cd} / \mathrm{m}^{2}\right.$ for REX) or a projector $\left(24 \mathrm{~cd} / \mathrm{m}^{2}\right.$ for Tempo). The fixation point and peripheral stimuli were filled circles with $0.4^{\circ}$ and $0.7^{\circ}$ diameters in the Tempo/Win system. The appearance and disappearance of visual stimuli were synchronized with the projector's vertical refresh (noninterlaced refresh rate of $60 \mathrm{~Hz}$ ).

Behavioral paradigm. Each trial was preceded by a $600 \mathrm{~ms}$ intertrial interval during which the screen was illuminated with a diffuse light to prevent dark adaptation. After the removal of the background light, a fixation point appeared in the center of the screen and the monkeys were required to direct their eyes toward the fixation point within $30 \mathrm{~s}$. After they had maintained steady fixation for 900-1200 $\mathrm{ms}$ (5 fixation lengths with equal steps) within a computer-controlled window $\left( \pm 2-3.5^{\circ}\right)$, a red stimulus was presented either $15^{\circ}$ left or right from the fixation point and the monkeys generated a saccade either toward the stimulus (prosaccade) or in the opposite direction of the stimulus (antisaccade) within $600 \mathrm{~ms}$ based upon fixation point color (red: pros; green: anti) (Fig. $1 \mathrm{~A}$ ). An additional $150-350 \mathrm{~ms}$ of fixation was required on the red stimulus on prosaccade trials or on a green stimulus presented at the mirror position of the red stimulus after saccade initiation on antisaccade trials. The size of fixation windows for peripheral

\section{Materials and Methods}

General. All experimental procedures were conducted in accordance with the Canadian Council on Animal Care policy on the use and care of laboratory animals and approved by the Queen's University Animal Care Committee. Surgical and electrophysiological procedures were described previously (Marino et al., 2008). Briefly, two male monkeys (Macaca mulatta), weighing 13.5 and $10 \mathrm{~kg}$, were implanted with scleral search coils, a head-restraining device, and a recording chamber under gaseous isofluorene $(2-2.5 \%)$ anesthesia with the analgesic buprenorphine (0.01-0.02 mg/kg i.m.). Horizontal and vertical eye positions were sampled at $1 \mathrm{kHz}$ using the search coil technique (Robinson, 1963; Fuchs and Robinson, 1966; Judge et al., 1980). The onset and end of saccades were identified by radial eye velocity criteria (threshold: $30 \%$ ). The recording chamber (circular, $19 \mathrm{~mm}$ inner diameter, tilted by $34^{\circ}$ laterally and $13^{\circ}$ anteriorly in monkey $\mathrm{O}$ and $36^{\circ}$ laterally in monkey E) was placed on the left hemisphere in both monkeys to cover the head and body of the caudate nucleus. Using the grid system (Crist et al., 1988), we mapped the caudate nucleus as widely as possible in the area allowed by each chamber. Recording sites were verified by magnetic resonance imaging (MRI) ( 3 tesla; Siemens) in one monkey (monkey O), whose implant was compatible with MRI (Fig. 1C). Neurons with fewer than five trials in each condition were excluded from analyses. All quantitative analyses for single neuron recordings were performed by calculating firing rates using spike counts within temporal windows associated with task events. Several characteristics of firing rates unfavorable for parametric analyses (Dean, 1981; Tolhurst et al., 1981) were corrected by taking their square root (Prince et al., 2002). We confirmed similar results without this correction.

Experimental systems. Single neuron recordings were performed using the Plexon data acquisition system. We isolated single neurons online, stimuli were $\pm 10^{\circ}$ to accept relatively inaccurate antisaccades (Bell et al., 2000). The monkeys occasionally generated corrective saccades toward the green stimulus after correct antisaccades were terminated, although corrective saccades were not required as long as eye positions were within the fixation window. We confirmed major results reported in this study when trials with corrective saccades were excluded from analyses. The monkeys received a liquid reward after each correct trial. On the half of the trials, the fixation point remained visible until the end of each trial (Fig. 1 B, overlap condition). On the remaining half of the trials, a $200 \mathrm{~ms}$ gap was introduced between fixation point disappearance and stimulus appearance (gap condition). For the majority of data analyses described here, we focus on the overlap condition (see Dataset below). The pro/anti instructions, left/right stimulus locations, and gap/overlap conditions were randomly interleaved in the block of trials.

Definition of presaccade and perisaccade periods. We defined a presaccade ( -90 to $-30 \mathrm{~ms}$ from saccade initiation) and perisaccade $(-30$ to $+30 \mathrm{~ms}$ from saccade initiation) period to characterize the saccade direction selectivity of individual caudate neurons. We first determined the delay of caudate neurons for saccade control based on several previous findings. Microstimulation of caudate neurons induces inhibitory and excitatory responses in the substantia nigra pars reticulata $(\mathrm{SNr})$ with an average latency of $16.7 \mathrm{~ms}$ for both responses (Hikosaka et al., 1993). The latencies of antidromic activation from the superior colliculus (SC) to the SNr vary from 0.7 to $2.3 \mathrm{~ms}$ (Hikosaka and Wurtz, 1983). Microstimulation of SC neurons during saccades influences saccade trajectories with a minimum latency of 8-10 ms (Miyashita and Hikosaka, 1996; Munoz et al., 1996). Therefore, the latency of caudate neurons to influence eye movements could be as little as $26 \mathrm{~ms}$. For simplicity, we used a $30 \mathrm{~ms}$ delay for our caudate neurons. Prosaccade and antisaccade durations 
were $51 \pm 11$ and $58 \pm 13 \mathrm{~ms}$ (mean $\pm \mathrm{SD}$ ), respectively. Therefore, we took $60 \mathrm{~ms}$ as a time for saccade duration for simplicity and defined the perisaccade period as between -30 and $30 \mathrm{~ms}$ from saccade initiation and the presaccade period as between -90 and $-30 \mathrm{~ms}$ from saccade initiation. We confirmed our results using another $60 \mathrm{~ms}$ time window starting $15 \mathrm{~ms}$ earlier than the presaccade period to take into account a potential delay for the indirect pathway compared with the direct pathway (Tachibana et al., 2008).

Definition of prestimulus period. We defined a prestimulus period ( $0-80 \mathrm{~ms}$ after stimulus appearance) by estimating the shortest visual latency of caudate neurons after stimulus appearance. We compared activity between contralateral and ipsilateral saccade trials in each instruction (pro/anti) separately by two-tailed $t$ tests $(p<0.05)$ with a 60 $\mathrm{ms}$ temporal window shifting by $10 \mathrm{~ms}$ within $600 \mathrm{~ms}$, starting $100 \mathrm{~ms}$ before stimulus appearance. Because $t$ tests detected occasional statistical significances before $50 \mathrm{~ms}$ after stimulus appearance at which only $5 \%$ of neurons in cortical regions projecting to the caudate nucleus show visual responses (Schmolesky et al., 1998), we also calculated the following index (DeAngelis and Uka, 2003) shown in Equations 1 and 2:

$$
\begin{gathered}
\text { Saccade direction index }=\frac{C-I}{|C-I|+2 R M S_{\text {error }}} \\
R M S_{\text {error }}=\sqrt{S S E /(N-M)}
\end{gathered}
$$

where $C$ and $I$ denote the average firing rates on contralateral and ipsilateral saccade trials, respectively. $R M S_{\text {error }}$ is defined by Equation 2. $\mathrm{N}$ is the total number of trials. $M$ is the number of conditions (2). The absolute value of this index is close to 1 when the difference between the average firing rates for contralateral and ipsilateral saccades is much larger than the variance in firing rates, while it is close to 0 when the variance is dominant over the difference. Positive and negative saccade direction indices indicate contralateral and ipsilateral saccade direction preferences, respectively. We identified a set of criteria for saccade direction indices to exclude inappropriate statistical detections (5 consecutive time points exceeding 0.17 and the maximum value reaching at least 0.33 before falling below 0.17 ; when a saccade direction index is equal to 0.33 , the absolute difference between contralateral and ipsilateral saccades is equal to the $R M S_{\text {error }}$ ). We detected the earliest saccade direction discrimination at $80 \mathrm{~ms}$ after stimulus appearance. Shorter temporal windows detected equal or longer latencies. Therefore, we adopted this $80 \mathrm{~ms}$ time window starting at stimulus appearance as the prestimulus period.

Definition of task-related neurons. In all analyses reported in this study, trials with reaction times shorter than $110 \mathrm{~ms}$ were excluded. This criterion for the shortest reaction times was defined to avoid the potential contamination of prestimulus activity $(0-80 \mathrm{~ms}$ after stimulus appearance) with saccadic activity (30 ms delay for caudate neurons). This criterion excluded only 0.6 and $3.1 \%$ of correct and direction error trials, respectively, compared with when the criterion was set to $70 \mathrm{~ms}$.

We adopted the same definition of task-related neurons that we used in a previous report (Watanabe and Munoz, 2009). For the definitions of task-related neurons and their preferred saccade directions, we collapsed trials with the overlap and gap conditions because it allowed us to classify more neurons by increasing the number of trials for reliable statistical outcome. We confirmed results focusing only on the overlap conditions when we performed this neuron classification using the overlap condition only. We calculated firing rates during the following four periods: $120-180 \mathrm{~ms}$ after stimulus appearance, $180-240 \mathrm{~ms}$ after stimulus appearance, -90 to $-30 \mathrm{~ms}$ from saccade initiation (presaccade period), and -30 to $+30 \mathrm{~ms}$ from saccade initiation (perisaccade period). The former two periods were defined for visual responses (peak visual responses were observed $\sim 180 \mathrm{~ms}$ after stimulus appearance) (see Fig. $5 A, C)$. We identified the best window to maximize a difference between two of the four conditions [ 2 instructions (pro and anti) $\times 2$ saccade directions (contra and ipsi) \{rsqb] using Equation 3, which is similar to the saccade direction index:

$$
\text { Discrimination index }=\frac{C_{\max }-C_{\min }}{C_{\max }-C_{\min }+2 R M S_{\text {error }}},
$$

where $C_{\max }$ and $C_{\min }$ indicate the maximum (max) and minimum (min) average firing rates among the four conditions, respectively. $R M S_{\text {error }}$ is defined by Equation 2. We performed a two-way ANOVA (main factors: instructions and saccade directions, $p<0.05$ ). In addition, we compared the maximum average firing rates $\left(C_{\max }\right)$ and firing rates during the prestimulus period using a one-tailed $t$ test $(p<0.05)$. For calculation of the prestimulus activity, we chose trials whose instruction was the same as that associated with the $C_{\max }$ and collapsed trials with opposite saccade directions, because prestimulus activity was not different before caudate neurons responded to stimulus appearance. We considered neurons as task-related if both the two-way ANOVA and $t$ test detected statistical significance. Bonferroni correction was applied to the statistical tests described above to take into account multiple temporal windows.

Secondary criteria for task-related neurons. The criteria of task-related neurons described above detect neurons whose activity was modulated by the behavioral paradigm after stimulus appearance. However, it was possible that several caudate neurons showed activity modulated by a task instruction (pro or anti) given by the color of the fixation point before stimulus appearance. To identify such neurons, we adopted the following criteria. We first collapsed trials with opposite saccade directions, because stimulus information was not yet available during the prestimulus period. We then compared firing rates on prosaccade and antisaccade trials during the prestimulus period ( $t$ test, $p<0.05$ ). To ensure that the instruction preferences were caused by increases in firing rates on trials with the preferred instruction, we compared firing rates during the prestimulus period and those during an $80 \mathrm{~ms}$ temporal window (same length as the prestimulus period) starting at $200 \mathrm{~ms}$ after fixation initiation on trials with the preferred instruction (we took the $200 \mathrm{~ms}$ delay to wait for eye positions stabilized) (one tailed $t$ test, $p<$ 0.05 , divided by two to take into account two instructions). We assigned neurons as task-related neurons if they satisfied both statistical tests.

Definition of preferred saccade directions. We further classified taskrelated neurons detected by the criteria described above based on their saccade direction preferences. Figures 2 and 3 show three examples of saccade-related neurons in the caudate nucleus whose activity was aligned with stimulus appearance (Fig. 2) and saccade initiation (Fig. 3). Briefly, the first example neuron (Figs. $2 A, B, 3 A, B$ ) had stronger activity when a stimulus was presented on the contralateral side (contralateral prosaccade and ipsilateral antisaccade trials). The second example neuron (Figs. $2 C, D, 3 C, D$ ) had stronger saccadic activity when saccades were directed toward the contralateral direction. The third example neuron (Figs. $2 E, F, 3 E, F$ ) had saccadic activity specialized for ipsilateral antisaccades.

To define the saccade direction preferences of individual task-related neurons in the caudate nucleus quantitatively, we adopted the following criteria. For each instruction (prosaccade or antisaccade), we compared firing rates on contralateral and ipsilateral saccade trials during the presaccade and perisaccade periods by calculating the saccade direction index (Equation 1). We identified one of the two windows with larger absolute value of the saccade direction index for the following analyses. We defined neurons as discriminating saccade direction if they showed a significant difference between firing rates for contralateral and ipsilateral saccades on prosaccade and/or antisaccade trials $(t$ test, $p<0.05)$ and if firing rates for the preferred direction exceeded the prestimulus activity (one tailed $t$ test, $p<0.05$ ).

The definition of saccade direction preferences was straightforward when saccade direction preferences were the same on both prosaccade and antisaccade trials in individual neurons (Fig. $4 \mathrm{~A}$, quadrants I and III) (for example see Figs. $2 C, D, 3 C, D$ ). When saccade direction preferences were the opposite between prosaccade and antisaccade trials (Fig. $4 \mathrm{~A}$, quadrants II and IV), we compared prosaccade and antisaccade trials with their own preferred directions by the same procedure described above. For instance, for neurons with contralateral prosaccade and ipsi- 

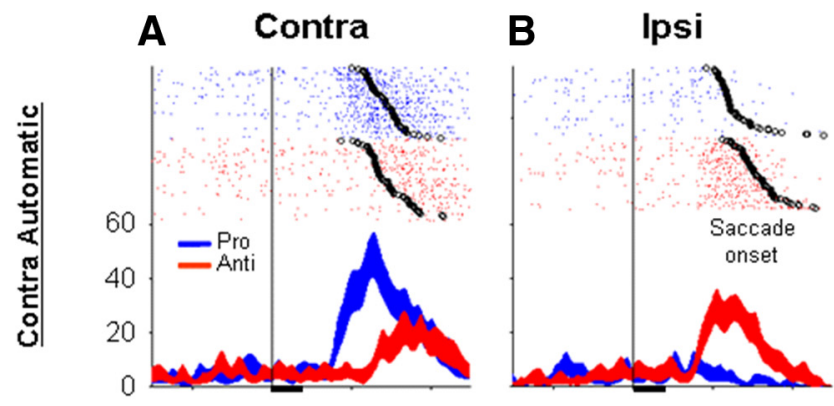

C

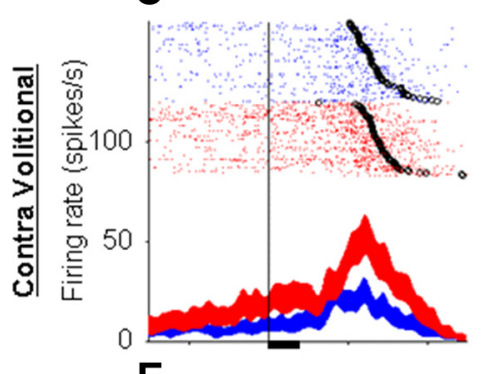

E

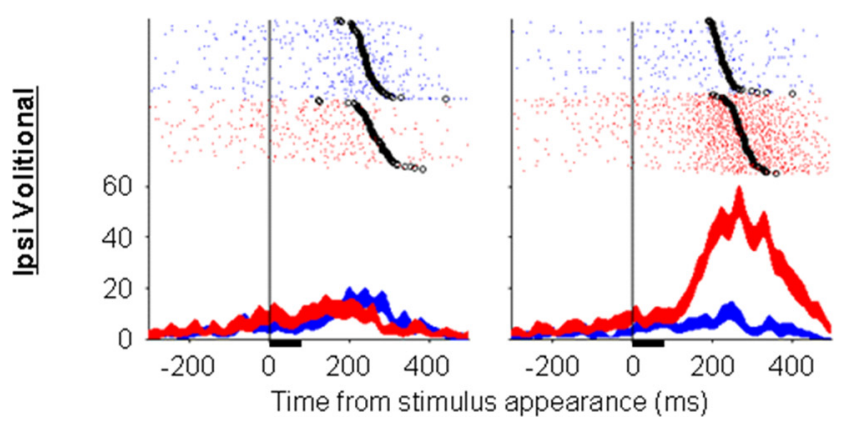

Figure 2. Activity of individual saccade-related neurons in caudate nucleus aligned with stimulus appearance (overlap condition). $\boldsymbol{A}, \boldsymbol{B}$, Automatic neuron with contralateral (Contra) saccade preference. $\boldsymbol{C}, \boldsymbol{D}$, Volitional neuron with contralateral saccade preference. $\boldsymbol{E}, \boldsymbol{F}$, Volitional neuron with ipsilateral (Ipsi) saccade preference. Left $(\boldsymbol{A}, \boldsymbol{C}, \boldsymbol{E})$ and right $(\boldsymbol{B}, \boldsymbol{D}, \boldsymbol{F})$ columns indicate contralateral and ipsilateral saccade trials, respectively. Black circles indicate saccade initiation. Black horizontal bars under the $x$-axes indicate the prestimulus period $(0-80 \mathrm{~ms}$ after stimulus appearance). Data from the overlap condition are shown.

lateral antisaccade preferences (Fig. $4 A$, quadrant IV) (for example, see Figs. $2 A, B, 3 A, B$ ), we compared firing rates on contralateral prosaccade trials and ipsilateral antisaccade trials and defined its saccade direction preference as contralateral (or ipsilateral) if firing rates were higher on contralateral prosaccade (or ipsilateral antisaccade) trials.

Several neurons showed saccade direction preferences only in one of the two instructions (pro or anti). For these neurons, we applied the same procedure for neurons with opposite saccade direction preferences described above. For instance, if a neuron showed a ipsilateral saccade direction preference on antisaccade trials (for example, see Figs. $2 E, F$, $3 E, F)$, we defined its saccade direction preference as ipsilateral given that firing rates during antisaccades toward the preferred (ipsilateral) direction were higher than those during prosaccades toward the opposite (contralateral) direction. This excludes a possibility that the strongest activity of this neuron was associated with saccades toward the opposite of the antisaccade-preferred direction. Bonferroni correction was applied to the classification criteria described so far to take into account the multiple comparisons as well as multiple temporal windows.

Dataset. Using the above criteria, we identified 111 task-related neurons (monkey O: 72; monkey E: 39) among 277 neurons (monkey O: 162; monkey E: 115) that we encountered while monkeys performed the behavioral paradigm. The rate of task-related neurons (40\%) was slightly higher than that in the original report (34\%) (Hikosaka et al., 1989a),
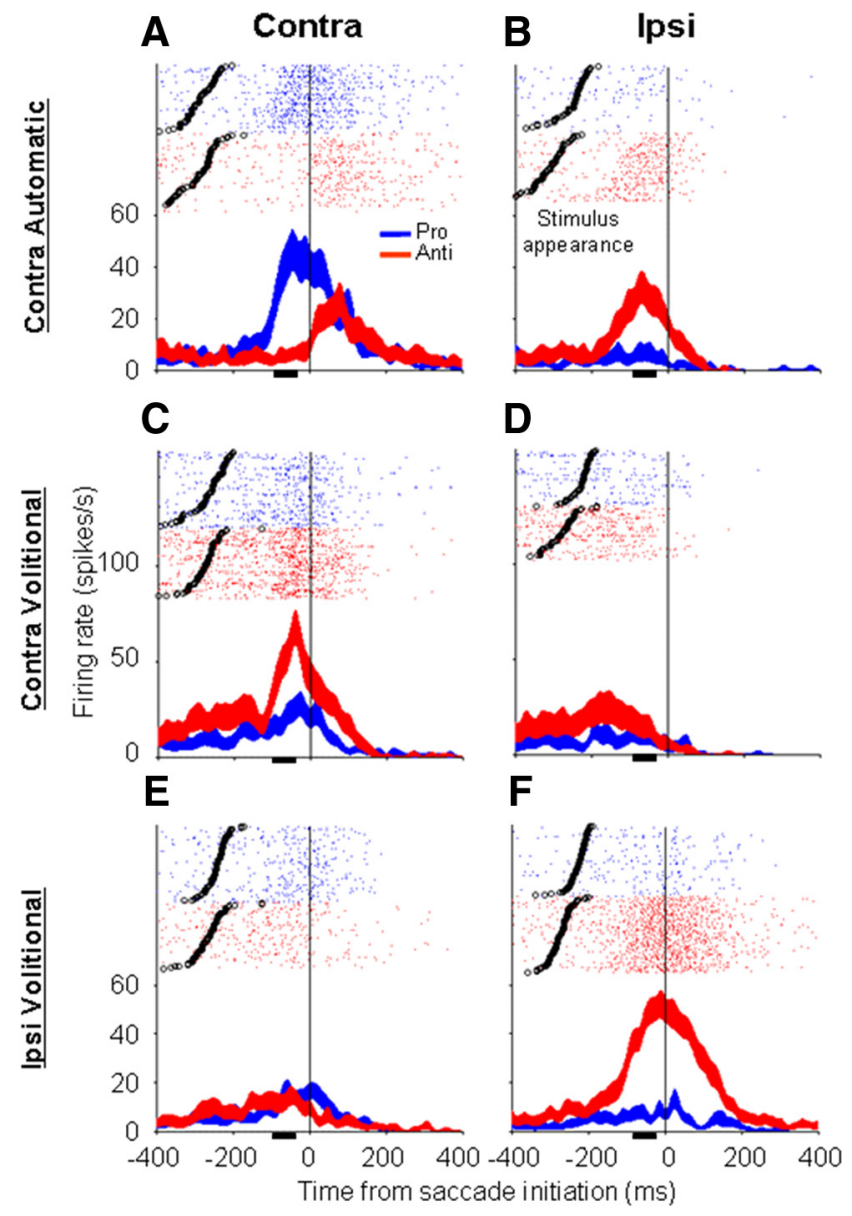

Figure 3. Activity of the same neurons shown in Figure 2 aligned with saccade initiation $(\boldsymbol{A}-\boldsymbol{F})$. Black circles indicate stimulus appearance. Black horizontal bars under the $x$-axes indicate the presaccade period ( -90 to $-30 \mathrm{~ms}$ from saccade initiation).

presumably because of our online selection of task-related neurons. We identified three task-related neurons in this population (monkey O: 2; monkey E: 1) whose activity was modulated only by task instructions (pro or anti) before stimulus appearance by secondary criteria.

Among the 111 task-related neurons, 67 neurons (monkey O: 43; monkey E: 24) had contralateral saccade direction preferences (Fig. $4 \mathrm{~A}$, $\mathrm{CN}$ ), while 20 neurons (monkey O: 12, monkey E: 8) had ipsilateral saccade direction preferences (Fig. $4 A$, IN). Eleven neurons (monkey O: 8; monkey E: 3) did not show saccade direction preferences either on prosaccade or antisaccade trials (Fig. $4 A, \mathrm{NN}$ ). There were 13 neurons (monkey O: 9; monkey E: 4) that showed saccade direction preferences on prosaccade and/or antisaccade trials but were not classified as $\mathrm{CNs}$ or INs by the above criteria (Fig. $4 \mathrm{~A}, \mathrm{UN}$ ). UNs did not have a significant influence on our results because we confirmed similar results when we assigned three UNs (monkey O: 1; monkey E: 2) in quadrant I in Figure $4 A$ as CNs and 10 UNs (monkey O: 8; monkey E: 2) in quadrant IV as CNs or INs.

The numbers of neurons with contralateral and ipsilateral saccade direction preferences were not different between two monkeys $\left(\chi^{2}\right.$ test, $\left.\mathrm{df}=1, \chi^{2}=0.12, p>0.7\right)$. When we classified contralateral saccade- and ipsilateral saccade-preferred neurons further into automatic and volitional neurons based on their presaccade activity on antisaccade trials (Fig. $4 B$ ) (see Results for details), their numbers were not different between two monkeys (Table $1, \mathrm{df}=3, \chi^{2}=1.9, p>0.6$ ). Thirty-five ( 12 automatic and 23 volitional neurons) of the 87 neurons with contralateral and ipsilateral saccade direction preferences were recorded by the REX system, and the remaining neurons (11 automatic and 42 volitional neurons) were recorded by the Tempo/Win system. Recording sites were not different between contralateral saccade- and ipsilateral saccade- 

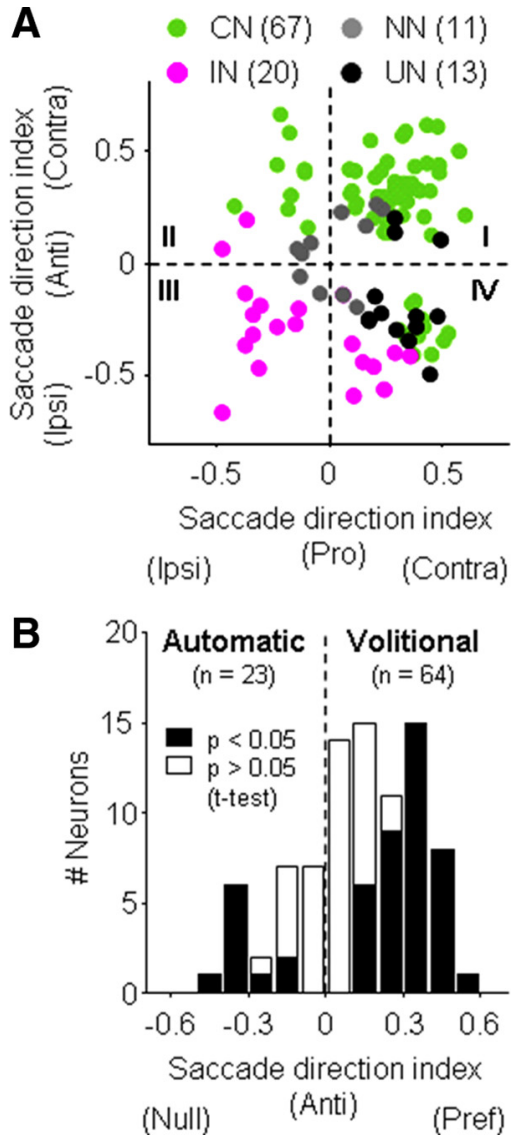

Figure 4. Neuron classification. $\boldsymbol{A}$, Classification of task-related neurons based on saccade direction preferences (overlap and gap conditions). CN, Contralateral (Contra) saccade preferred neurons; IN, ipsilateral (Ipsi) saccade preferred neurons; NN, nonsaccade-related neurons; UN, unclassified neurons. For each neuron, saccade direction indices were calculated during either the presaccade $(-90$ to $-30 \mathrm{~ms}$ from saccade initiation) or perisaccade $(-30$ to $+30 \mathrm{~ms}$ from saccade initiation) period. Saccade direction indices with larger absolute values were chosen for this neuron classification. $\boldsymbol{B}$, Saccade direction indices on antisaccade (Anti) trials during the presaccade period (overlap condition). Black bars indicate neurons with statistical significance ( $t$ test, $p<0.05$ ). For ipsilateral saccade-preferred neurons, the signs of saccade direction indices were flipped. Accordingly, the positive and negative values of saccade direction indices indicate that neurons had higher and lower presaccade activity, respectively, when antisaccades were directed toward their preferred direction (Pref) in both contralateral saccade- and ipsilateral saccade-preferred neurons. We classified these neurons further based on the signs of their indices (negative: automatic neurons, positive: volitional neurons).

Table 1. Neuron classification

\begin{tabular}{llcl}
\hline & Contra & Ipsi & Total \\
\hline Automatic & $19(10)$ & $4(2)$ & $23(12)$ \\
Volitional & $48(33)$ & $16(10)$ & $64(43)$ \\
Total & $67(43)$ & $20(12)$ & $87(55)$ \\
\hline
\end{tabular}

We classified saccade-related neurons in the caudate nucleus based on contralateral (Contra)/ipsilateral (Ipsi) saccade direction preferences and automatic/volitional encodings. See Materials and Methods for the definitions of contralateral and ipsilateral saccade direction preferences and Results for the definitions of automatic and volitional encodings. Numbers in parentheses indicate neurons from monkey 0 .

preferred neurons or between those encoding automatic and volitional saccades (Fig. 1, $D$ and $E$ for monkey $\mathrm{O}$ and E, respectively) (two-way ANOVA with the main factors of contra/ipsi preferences and automatic/ volitional encodings applied to each dimension: anterior-posterior, lateral-medial, dorsa-ventral, $\mathrm{df}=1, F<3.8, p>0.05)$. The firing characteristics of saccade-related neurons were consistent with phasically active neurons reported previously because of their phasic activity after stimulus appearance (Watanabe and Munoz, 2009) and low firing rates during fixation $(200-700 \mathrm{~ms}$ after fixation initiation, mean $\pm \mathrm{SD}=$
$2.4 \pm 2.2$ spikes/s, no difference between different types of neurons, two-way ANOVA, with the main factors of contra/ipsi preferences and automatic/volitional encodings, $\mathrm{df}=1, F<2.6, p>0.1$ ) (Kimura et al., 1984; Hikosaka et al., 1989a; Wilson et al., 1990; Aosaki et al., 1995; Apicella, 2007).

We focus on the overlap condition only in the description of the results, because the buildup activity of caudate neurons could be disrupted or contaminated by signals in response to fixation point disappearance before stimulus appearance in the gap condition. Nevertheless, we compared the buildup activity of caudate neurons between overlap and gap conditions in 52 neurons (see Fig. 5 legend for the number of neurons in each category). During the recordings of these neurons, total fixation durations were equal for the overlap and gap conditions. In the remaining neurons excluded from this analysis, total fixation durations were 200 ms longer in the gap condition compared with the overlap condition.

Multiple linear regression analysis. The buildup activity of caudate neurons before they responded to stimulus appearance depended on multiple parameters. To quantify the influence of task instructions and elapsed time from fixation initiation on the buildup activity of caudate neurons, we performed the following multiple linear regression analyses in individual neurons as shown in Equation 4,

$$
\text { Firing rate }=b_{i} \times[\text { task instruction }]+b_{f} \times[\text { fixation duration }] \text {, }
$$

where "task instruction" indicates prosaccade $(-1)$ and antisaccade $(+1)$ instructions, "fixation duration" indicates a time period from fixation initiation (when monkeys acquired the fixation point) to stimulus appearance, and "firing rate" was calculated during the prestimulus period. Firing rates and fixation durations were normalized by their means and SDs before applying this equation. We collapsed trials with opposite saccade directions for this analysis, because the prestimulus activity was not different between trials with opposite saccade directions. Because foveation of the fixation point was usually acquired by saccades, fixation duration was recalculated from the end of the saccade to stimulus appearance during offline analysis. To quantify the effect of a fixation gap, we performed the same multiple linear regression analysis with an additional factor (gap: +1 ; overlap: -1 ).

We did not find significant differences in regression coefficients (see Figs. 6, 7) between neurons recorded by the two experimental systems $(t$ test, $p>0.2)$ except for the regression coefficients of task instructions in automatic neurons ( $\mathrm{df}=21, t=-2.53, p<0.05$ ), which showed a negative bias (prosaccade instruction preferences) in neurons recorded by the REX system ( $\mathrm{df}=11, t=-2.21, p<0.05)$, but not in those recorded by the Tempo/Win system $(\mathrm{df}=10, t=1.42, p>0.1)$.

Correlation with reaction times. To examine whether the buildup activity of caudate neurons before stimulus appearance contributed to behavioral control, we analyzed relationships between reaction times and firing rates on a trial-by-trial basis. We calculated Pearson's partial correlation coefficients between reaction times and firing rates during the prestimulus period with fixation duration and other saccade parameters (peak velocity, duration, and horizontal and vertical amplitude) fixed to exclude their potential contributions to both reaction times and firing rates.

To examine the influence of spatial information on the correlation between reaction time and caudate activity, we performed the same analysis described above by using firing rates calculated during temporal periods (120-180 ms after stimulus appearance on average) defined by the following procedure. We created a cumulative reaction time distribution in each condition (instructions $\times$ saccade directions $\times$ monkeys $\times$ systems, 16 conditions), determined a time point at which the cumulative distribution exceeded $5 \%$, and then defined the temporal period for a $60 \mathrm{~ms}$ temporal window ended at $30 \mathrm{~ms}$ before the $5 \%$ time point. We did not find significant differences in correlation coefficients (see Fig. 8) between neurons recorded by the two experimental systems, ( $t$ test $p>0.2)$.

Direction error analysis. To compare activity between correct antisaccades and direction errors (making saccades toward the stimulus) in 
response to the same stimulus, we performed the following multiple linear regression analysis shown in Equation 5,

Firing rate $=b_{r} \times[$ behavioral response $]+b_{f} \times[$ fixation duration],

where "behavioral response" indicates correct $(+1)$ and direction error $(-1)$ responses and "fixation duration" indicates a time period from fixation initiation to stimulus appearance (Fig. $1 B$ ). We took into account fixation durations to exclude their potential influences on the comparison between correct and direction error trials, although fixation durations were not different between the two types of trials at the population level ( $t$ test, $p>0.3$ ). We did not find significant differences in regression coefficients (see Fig. 9) between neurons recorded by the two experimental systems ( $t$ test, $p>0.2$ ).

\section{Results}

\section{Behavior}

The behavior of our monkeys was consistent with previous reports in both humans (Hallett, 1978; Fischer and Weber, 1992; Dafoe et al., 2007) and monkeys (Bell et al., 2000; Watanabe and Munoz, 2009). Antisaccade reaction times were longer than prosaccade reaction times (overlap condition, average reaction times \pm SD in monkey O: pro, $268 \pm 65 \mathrm{~ms}$, anti, $299 \pm 62 \mathrm{~ms}, t$ test: $\mathrm{df}=15762, t=30.6, p<0.0001$; overlap condition, average reaction times $\pm \mathrm{SD}$ in monkey E: pro, $282 \pm 55 \mathrm{~ms}$, anti, $320 \pm$ 75 ms, $t$ test: $\mathrm{df}=9024, t=28.1, p<0.0001)$. Direction error rates were higher on antisaccade trials compared with prosaccade trials (overlap condition in monkey O: pro, 3.6\%, anti, $12.8 \%, \chi^{2}$ test: $\mathrm{df}=1, \chi^{2}=475, p<0.0001$; overlap condition in monkey E: pro, $1.6 \%$, anti, $2.8 \%, \chi^{2}$ test: $\left.\mathrm{df}=1, \chi^{2}=14.8, p<0.0005\right)$. These behavioral effects were observed in both recording systems ( $t$ test results for reaction times in monkey O in REX: $t=22.2$, $\mathrm{df}=7110, p<0.0001 ; t$ test results for reaction times in monkey O in Tempo: $t=21.4$, df $=8650, p<0.0001 ; t$ test results for reaction times in monkey $\mathrm{E}$ in REX: $t=22.7$, df $=4293, p<$ 0.0001 ; $t$ test results for reaction times in monkey $\mathrm{E}$ in Tempo: $t=$ $22.2, \mathrm{df}=4165, p<0.0001 ; \chi^{2}$ test results for direction error rates in monkey $\mathrm{O}$ in REX: $\chi^{2}=206, \mathrm{df}=1, p<0.0001 ; \chi^{2}$ test results for direction error rates in monkey O in Tempo: $\chi^{2}=282$, df $=1, p<0.0001 ; \chi^{2}$ test results for direction error rates in monkey E in REX: $\chi^{2}=4.25, \mathrm{df}=1, p<0.05 ; \chi^{2}$ test results for direction error rates in monkey $\mathrm{E}$ in Tempo: $\chi^{2}=11.5, \mathrm{df}=1$, $p<0.001)$.

\section{Caudate neurons encoding automatic and volitional saccade commands}

Among 277 neurons we encountered while monkeys performed the behavioral paradigm, we identified 87 saccade-related neurons in the caudate nucleus (see Dataset in Materials and Methods). We have reported previously that there are different types of saccade-related caudate neurons that encode either automatic or volitional saccade commands predominantly (Watanabe and Munoz, 2009). Figures 2 and 3 show the activity of three example caudate neurons whose activity was aligned with stimulus appearance (Fig. 2) and saccade initiation (Fig. 3). The first example neuron (Figs. $2 A, B, 3 A, B$ ) had stronger activity when a stimulus was presented on the contralateral side (contralateral prosaccade and ipsilateral antisaccade trials), suggesting that it responded to stimulus appearance and/or encoded predominantly automatic saccades toward the contralateral stimulus. This neuron also increased activity on contralateral antisaccade trials, but the activity occurred only after saccade initiation (Fig. $3 A$ ). The second example neuron (Figs. $2 C, D, 3 C, D$ ) had saccadic activity when sac- cades were directed toward the contralateral direction on both prosaccade and antisaccade trials, suggesting that it encoded predominantly contralateral volitional saccades. The third example neuron (Figs. 2E, F, 3E, F) had similar characteristics with the second example neuron, except that its activity was rather specialized for antisaccades toward the ipsilateral direction.

As described in the Introduction, the simple requirement of the antisaccade paradigm (look away from the stimulus) dissociates an erroneous automatic saccade command toward the stimulus and a correct volitional saccade command in the opposite direction of the stimulus. To examine which saccade command (automatic or volitional) individual saccade-related neurons in the caudate nucleus preferred before antisaccade initiation, we calculated saccade direction indices (Equation 1 in Materials and Methods) during the presaccade period ( -90 to $-30 \mathrm{~ms}$ from saccade initiation indicated by black horizontal bars under $x$-axes in Fig. 3; see Definition of presaccade and perisaccade periods in Materials and Methods) on antisaccade trials (Fig. 4B). For ipsilateral saccade-preferred neurons (Figs. $2 E, F, 3 E, F$ ), we flipped the signs of saccade direction indices. Therefore, the positive and negative values of saccade direction indices in Figure $4 B$ indicate that neurons had higher presaccade activity when antisaccades were directed toward their preferred and null direction, respectively, in both contralateral and ipsilateral saccade-preferred neurons.

Neurons with negative saccade direction indices on antisaccade trials $(n=23)$ (Fig. $4 B)$, such as the first example neuron (Figs. $2 A, B, 3 A, B$ ), had higher activity when a stimulus was presented on their preferred side and an antisaccade was directed to their null direction (Fig. $5 A-D$, population spike density functions). They also increased activity slightly when antisaccades were directed toward their preferred direction, but this saccadic activity became dominant only after saccade initiation (Fig. $5 B, D$ ). These firing characteristics are consistent with our hypothesis that, before saccade initiation, neurons with negative saccade direction indices on antisaccade trials facilitate predominantly erroneous automatic saccades toward a stimulus presented on their preferred side (Watanabe and Munoz, 2009). These neurons might correspond to visual and/or visual contingent saccade neurons reported previously (Hikosaka et al., 1989a).

On the other hand, neurons with positive saccade direction indices on antisaccade trials $(n=64)$ (Fig. $4 B)$, such as the second (Figs. 2C,D, 3C,D) and third (Figs. $2 E, F, 3 E, F$ ) example neurons, had higher activity when an antisaccade was directed toward their preferred direction and a stimulus was presented on the opposite side (Fig. $5 E-H$ ). They also increased activity when saccades were directed toward their preferred direction on prosaccade trials (Fig. $5 E, F$ ), although the activity was weaker than that on antisaccade trials because of the existence of neurons specialized for antisaccades (Figs. $2 E, F, 3 E, F$ ). This characteristic is consistent with our hypothesis that neurons with positive saccade direction indices on antisaccade trials facilitate predominantly correct volitional saccades toward their preferred directions (Watanabe and Munoz, 2009). These neurons might correspond to the memory-contingent and/or visual and memorycontingent saccade neurons reported previously (Hikosaka et al., 1989a).

In the following analyses, we adopted the above classification criterion based on the signs (positive/negative) of saccade direction indices on antisaccade trials (Fig. $4 B$ ) to dissociate neurons presumably encoding erroneous automatic saccades toward the stimulus (negative indices, hereafter called automatic neurons) and those presumably encoding correct volitional saccades toward 


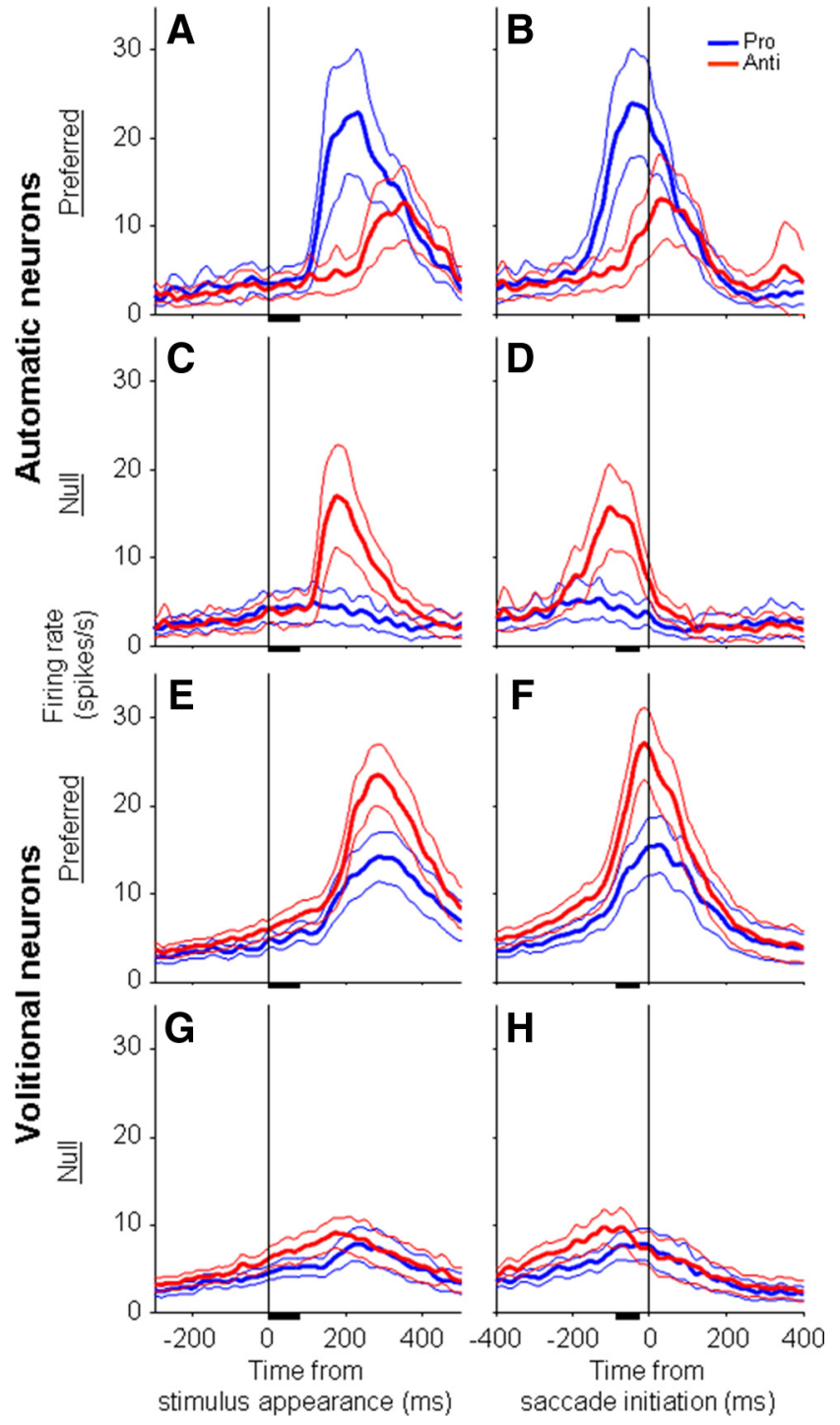

Figure 5. Population spike density functions (overlap condition). $\boldsymbol{A}-\boldsymbol{D}$, Automatic neurons ( $n=$ 23) on trials with saccades toward their preferred $(\boldsymbol{A}-\boldsymbol{B})$ and null $(\boldsymbol{C}, \boldsymbol{D})$ directions. $\boldsymbol{E}-\boldsymbol{H}$, volitional neurons $(n=64)$ on trials with saccades toward their preferred $(\boldsymbol{E}, \boldsymbol{F})$ and null $(\boldsymbol{G}, \boldsymbol{H})$ directions. Left $(\boldsymbol{A}, \boldsymbol{C}, \boldsymbol{E}, \boldsymbol{G})$ and right $(\boldsymbol{B}, \boldsymbol{D}, \boldsymbol{F}, \boldsymbol{H})$ columns show activity aligned with stimulus appearance and saccade initiation, respectively. Thick lines indicate population averages. Thin lines indicate $95 \%$ confidence interval. Black horizontal bars under the $x$-axes indicate the prestimulus period $(0-80 \mathrm{~ms}$ after stimulus appearance) in the left column $(A, C, E, G)$ and presaccade period ( -90 to $-30 \mathrm{~ms}$ from saccade initiation) in the right column $(\boldsymbol{B}, \boldsymbol{D}, \boldsymbol{F}, \boldsymbol{H})$. Pro, Prosaccade; Anti, antisaccade.

the opposite direction of the stimulus (positive indices, hereafter called volitional neurons). Although this criterion is rather qualitative, the statistical results described below were confirmed when we focused only on the subset of saccade-related neurons that showed significant saccade direction preferences on antisaccade trials (Fig. $4 B$, black bars). We analyzed neurons with contralateral and ipsilateral saccade preferences together because their firing characteristics were very similar (see supplemental Figs. 2-6, available at www. jneurosci.org as supplemental material).

\section{Selective activation of volitional neurons by antisaccade instruction}

As can be seen qualitatively in Figure 5, $E$ and $G$, volitional neurons presumably facilitating correct antisaccades toward their preferred direction had stronger activity on antisaccade trials compared with prosaccade trials even before caudate neurons responded to stimulus appearance. In contrast, such enhanced activity on antisaccade trials was not observed in automatic neurons presumably facilitating erroneous saccades toward the stimulus presented on their preferred side (Fig. $5 A, C$ ). To quantify the effects of task instruction (pro/anti) on the buildup activity of saccade-related caudate neurons before they responded to stimulus appearance, we performed multiple linear regression analyses (main factors: task instructions and fixation durations) during the prestimulus period $[0-80 \mathrm{~ms}$ after stimulus appearance indicated by black horizontal bars under $x$-axes (Fig. 2); see Definition of prestimulus period in Materials and Methods]. For this analysis, we collapsed trials with opposite saccade directions because the prestimulus activity of caudate neurons was not different between trials with opposite saccade directions. Positive values of regression coefficients for task instructions indicate higher activation on antisaccade trials compared with prosaccade trials.

For automatic neurons, the distribution of regression coefficients for task instructions was not biased toward either positive or negative values (Fig. $6 A$ ) ( $t$ test: $\mathrm{df}=22, t=-0.43, p>0.6$ ), indicating that they did not have task instruction preferences. In contrast, regression coefficients for task instructions of volitional neurons were biased toward positive values (Fig. $6 \mathrm{~B})(\mathrm{df}=63$, $t=5.2, p<0.0001)$, indicating their antisaccade instruction preferences. This result cannot be explained by differences in saccade parameters (endpoint, peak velocity, and duration) between prosaccade and antisaccades because we confirmed antisaccade instruction preferences in volitional neurons by taking into account the saccade parameters in multiple linear regression analyses (supplemental Fig. 1, available at www.jneurosci.org as supplemental material). We also found that regression coefficients for task instructions were correlated with saccade direction indices on antisaccade trials when automatic and volitional neurons were analyzed together (Pearson's $r=0.42, p<0.0001, n=87$ ). These results indicate that the prestimulus activity of neurons encoding volitional saccade commands was facilitated selectively when antisaccades were required.

Because prosaccade and antisaccade instructions were given by fixation point color well before stimulus appearance, it was possible that the antisaccade instruction preferences in volitional neurons emerged immediately after monkeys acquired the fixation point. However, their antisaccade instruction preferences did not emerge immediately after fixation initiation (Fig. 6C), but instead developed gradually before stimulus appearance (Fig. $6 D$ ). We confirmed similar results when neurons with opposite saccade direction preferences were analyzed separately (see supplemental Figs. 3, 4, available at www.jneurosci.org as supplemental material). To explain these temporal dynamics of antisaccade instruction preferences, it is important to consider additional factors that we describe in the following sections.

\section{Prestimulus activity increased with elapsed time from fixation initiation}

It has been shown previously that saccade reaction times are shortened with elapsed time from fixation initiation (Pare and Munoz, 1996; Oswal et al., 2007). Indeed, we confirmed this behavioral phenomenon in our monkeys on both prosaccade and antisaccade trials (monkey O: pro: Pearson's $r=-0.18, n=$ 8287, $p<0.0001$, anti: $r=-0.28, n=7477, p<0.0001$; monkey E: pro: $r=-0.16, n=4573, p<0.0001$, anti: $r=-0.18, n=$ 4453, $p<0.0001)$. This phenomenon was confirmed in both experiment systems (monkey $\mathrm{O}$ in REX: pro: $r=-0.19, n=$ 3749, $p<0.0001$, anti: $r=-0.32, n=3363, p<0.0001$; monkey 

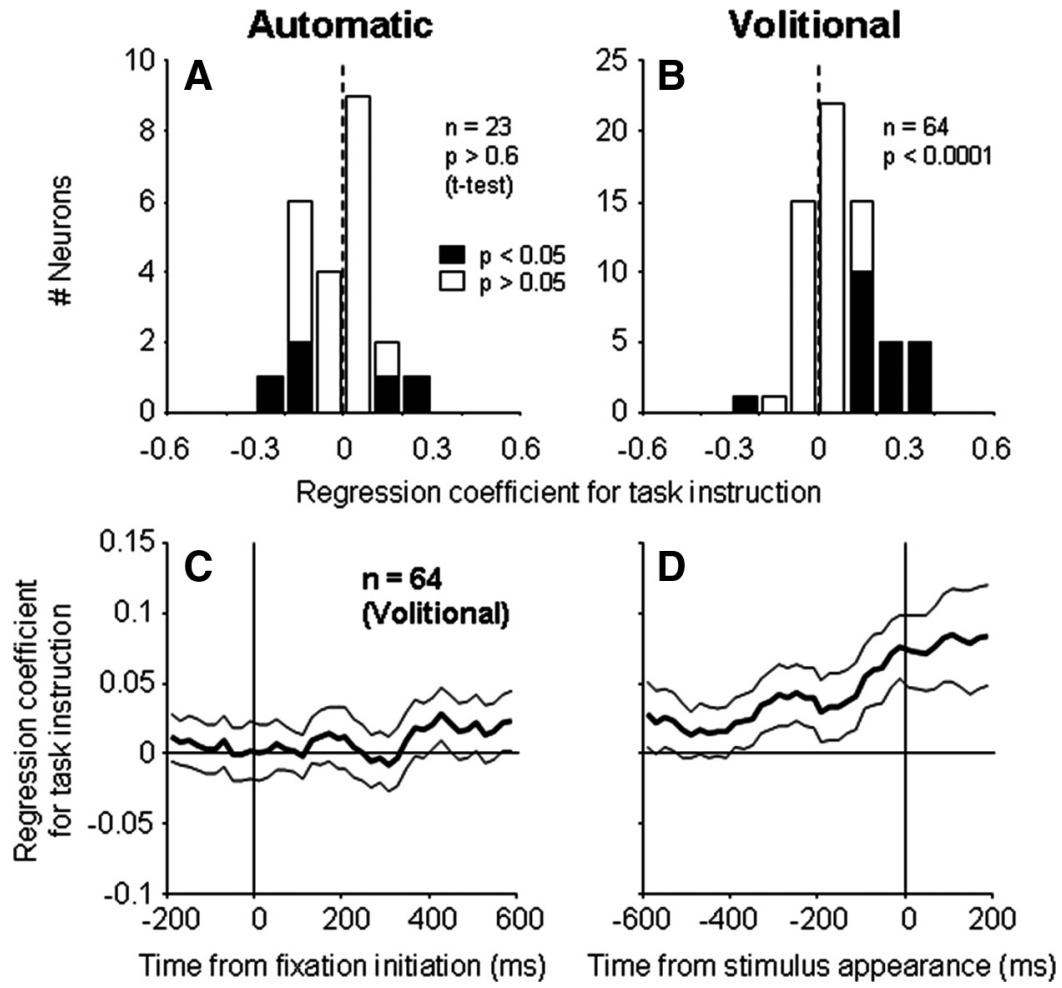

Figure 6. Task instruction dependence of caudate prestimulus activity (overlap condition). $A, B$, Regression coefficients for task instruction in automatic $(\boldsymbol{A})$ and volitional $(\boldsymbol{B})$ neurons. Positive and negative values of regression coefficients for task instruction indicate higher and lower prestimulus activity on antisaccade trials compared with prosaccade trials, respectively. Black bars indicate neurons with regression coefficients whose $95 \%$ confidence intervals did not include zero. C, D, Time course of regression coefficients for task instruction for volitional neurons (overlap condition) aligned with fixation initiation ( $\boldsymbol{C}$ ) and stimulus appearance (D). Thick lines indicate population averages. Thin lines indicate $95 \%$ confidence intervals.

Prestimulus activity was enhanced by a fixation gap

Saccade initiation is facilitated when a temporal gap is introduced between fixation point disappearance and stimulus appearance (gap effect) (Saslow, 1967). The neural correlates of the gap effect have been reported in the superior colliculus (Dorris and Munoz, 1995; Everling et al., 1998, 1999) and frontal eye field (Dias and Bruce, 1994; Everling and Munoz, 2000). If the prestimulus activity of automatic and volitional neurons in the caudate nucleus reflects preparatory set, it should increase during the fixation gap. Indeed, the example neuron shown in Figure 7, $A$ and $B$, had stronger prestimulus activity in the gap condition compared with the overlap condition on both prosaccade and antisaccade trials. To quantify this at the population level, we analyzed the prestimulus activity of 52 saccade-related neurons (see Fig. 7 legend for the numbers of neurons in each category) in which we used equal total fixation durations for the gap and overlap conditions (see Dataset in Materials and Methods).

We confirmed that our monkeys showed behavioral gap effects on both prosaccade and antisaccade trials (average reaction times $\pm \mathrm{SD}$ in monkey $\mathrm{O}$ : pro gap: $208 \pm 45 \mathrm{~ms}$, pro overlap: $264 \pm 66$ ms, $t$ test: $\mathrm{df}=8830, t=45.9, p<0.0001$, anti gap: $239 \pm 42 \mathrm{~ms}$, anti overlap: $293 \pm$

O in Tempo: pro: $r=-0.18, n=4538, p<0.0001$, anti: $r=$ $-0.24, n=4114, p<0.0001$; monkey E in REX: pro: $r=-0.10$, $n=2394, p<0.0001$, anti: $r=-0.18, n=2337, p<0.0001$; monkey E in Tempo: pro: $r=-0.23, n=2179, p<0.0001$, anti: $r=-0.18, n=2116, p<0.0001)$. If the prestimulus activity of automatic and volitional neurons in the caudate nucleus reflects preparatory set, it is expected to increase with elapsed time from fixation initiation.

Figure 7, $A$ and $B$, shows the activity of the same example neuron shown in Figures 2, $E$ and $F$, and $3, E$ and $F$. In this figure, trials with opposite saccade directions were collapsed. The buildup activity of this neuron started earlier relative to stimulus appearance when fixation durations were longer. To quantify the dependence of the prestimulus activity of automatic and volitional neurons on elapsed time from fixation initiation, we analyzed regression coefficients for fixation durations calculated by the same multiple linear regression analyses that quantified task instruction preferences (Fig. 6). For automatic neurons, the distribution of regression coefficients for fixation durations was biased toward positive values ( $\mathrm{df}=22, t=3.4, p<0.005$ ) (Fig. $7 C$ ), indicating that their prestimulus activity was higher when fixation durations were longer. We found the same effect for volitional neurons ( $\mathrm{df}=63, t=7.5, p<0.0001$ ) (Fig. $7 D)$. We confirmed similar results when neurons with opposite saccade direction preferences were analyzed separately (supplemental Fig. 4, available at www.jneurosci.org as supplemental material). These results indicate that elapsed time from fixation initiation is an important factor that the prestimulus activity of saccaderelated neurons in the caudate nucleus depended on.
$58 \mathrm{~ms}, t$ test: $\mathrm{df}=8045, t=47.6, p<0.0001$; average reaction times \pm SD in monkey E: pro gap: $202 \pm 50 \mathrm{~ms}$, pro overlap: $272 \pm 57 \mathrm{~ms}, t$ test: $\mathrm{df}=4102, t=41.7 p<0.0001$, anti gap: $234 \pm 58 \mathrm{~ms}$, anti overlap: $321 \pm 81 \mathrm{~ms}, t$ test: $\mathrm{df}=3946, t=38.1$, $p<0.0001)$.

To compare the prestimulus activity of saccade-related caudate neurons between the gap and overlap conditions, we performed the same multiple linear regression analyses performed for task instructions (Fig. 6) and fixation durations (Fig. 7C,D), with an additional factor for the gap and overlap conditions. We found that the distributions of regression coefficients for gap/ overlap conditions were biased toward positive values in both automatic ( $\mathrm{df}=10, t=2.4, p<0.05)$ (Fig. $7 E$ ) and volitional ( $\mathrm{df}=40, t=3.3, p<0.005)$ (Fig. $7 E$ ) neurons. This indicates that the prestimulus activity of automatic and volitional neurons was higher in the gap condition compared with the overlap condition. We confirmed similar results when neurons with opposite saccade direction preferences were analyzed separately (supplemental Fig. 4, available at www.jneurosci.org as supplemental material). We also confirmed that the prestimulus activity of volitional neurons in this population depended on task instructions ( $t$ test, $\mathrm{df}=40, t=3.7, p<0.001)$ and fixation durations $(\mathrm{df}=$ $40, t=5.4, p<0.0001)$ by analyzing regression coefficients calculated in this analysis at the same time, although the prestimulus activity of automatic neurons in this population did not show statistical significance in regression coefficients for task instructions $(\mathrm{df}=10, t=0.7, p>0.5)$ or fixation durations $(\mathrm{df}=10, t=$ $1.6, p>0.1)$. 
These results indicate that the fixation gap is another important factor on which the prestimulus activity of saccade-related neurons in the caudate nucleus depended.

\section{Correlation between prestimulus activity and behavior}

The analyses described so far examined the dependences of the prestimulus activity of saccade-related neurons in the caudate nucleus on task parameters, including task instructions (Fig. 6), elapsed time from fixation initiation (Fig. 7C,D), and fixation gap (Fig. $7 E, F$ ). The results are consistent with our hypothesis that the prestimulus activity reflects preparatory set and that the selective activation of volitional neurons by an antisaccade instruction presets BG circuits in favor of volitional saccades to compete against erroneous automatic saccades. To challenge this hypothesis further, we examined the correlation between the prestimulus activity of saccade-related caudate neurons and behavior by the following two analyses.

First, we calculated Pearson's partial correlation coefficients between prestimulus activity and saccade reaction times on a trial-by-trial basis with fixation duration and other saccade parameters fixed to exclude their potential contribution to both reaction times and firing rates. Automatic neurons had negative correlation at the population level when prosaccades were directed toward their preferred direction ( $t$ test: $\mathrm{df}=22, t=-2.4, p<0.05$ ) (Fig. $8 A$ ), indicating that reaction times were shorter when their prestimulus activity was higher. However, such negative correlation was not observed in the other task conditions $(\mathrm{df}=22,-1.3<t<0.7, p>$ 0.2 ) (Fig. $8 B-D$ ). Volitional neurons, on the other hand, had negative correlation for antisaccades toward both preferred $(\mathrm{df}=63, t=-5.4, p<0.0001)$ (Fig. 8G) and null $(\mathrm{df}=63, t=-2.8, p<0.05)$ (Fig. $8 H$ ) directions, but not for prosaccades toward either direction $(\mathrm{df}=63$, $|t|<1.2, p>0.2$ ) (Fig. $8 E, F)$. We confirmed this result when neurons with opposite saccade direction preferences were analyzed separately (supplemental Fig. 5, available at www.jneurosci.org as supplemental material). The negative correlation of volitional neurons for antisaccades toward both directions was mediated by a common set of volitional neurons, because volitional neurons with stronger negative correlation in one direction had similar strength of negative correlation in the opposite direction (Pearson's $r=0.29, p<0.05$ ). There was no difference between the correlation coefficients of volitional neurons for antisaccades toward the preferred and null directions (paired $t$ test, $\mathrm{df}=63, t=1.7, p>0.1$ ). The
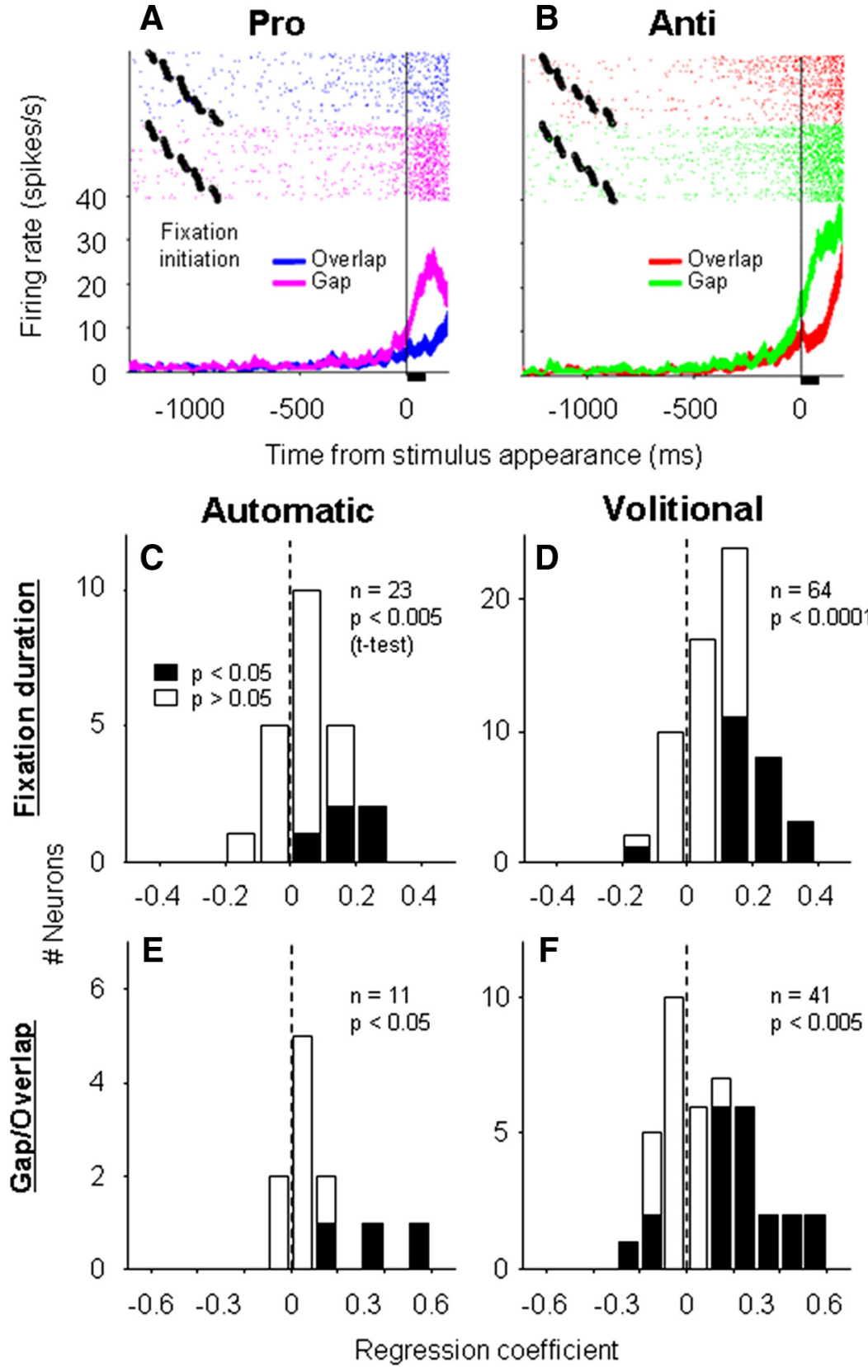

Figure 7. Fixation duration and fixation gap dependence of caudate prestimulus activity. $\boldsymbol{A}, \boldsymbol{B}$, The same volitional neuron shown in $E$ and $F$ in Figures 2 and 3 on prosaccade (Pro) $(\boldsymbol{A})$ and antisaccade (Anti) (B) trials. Trials with opposite saccade directions were collapsed. Rasters are sorted by fixation durations. Black horizontal bars under the $x$-axes indicate the prestimulus period (0-80 ms after stimulus appearance). C, D, Regression coefficients for fixation durations in automatic (C) and volitional (D) neurons (overlap condition). Positive and negative values of regression coefficients for fixation duration indicate that prestimulus activity increased and decreased with fixation durations, respectively. $\boldsymbol{E}, \boldsymbol{F}$, Regression coefficients for gap/overlap in automatic $(\boldsymbol{E})$ and volitional $(\boldsymbol{F})$ neurons. Positive and negative values of regression coefficients for gap/overlap indicate higher and lower prestimulus activity in the gap condition compared with the overlap condition, respectively. The numbers of neurons from each category are the following: Automatic-Contralateral, 9 (5); Automatic-Ipsilateral, 2 (1); Volitional-Contralateral: 34 (23); Volitional-Ipsilateral, 7 (4). Numbers in parentheses indicate neurons from monkey 0 . Black bars indicate neurons with regression coefficients whose $95 \%$ confidence intervals did not include zero.

negative correlation of volitional neurons became spatially specific after caudate neurons responded to stimulus appearance (average \pm SD of correlation coefficients calculated during $120 \sim 180 \mathrm{~ms}$ after stimulus appearance on average; preferred direction: $-0.22 \pm 0.16$, null direction: $-0.09 \pm$ 0.25 , paired $t$ test: $\mathrm{df}=63, t=4.3, p<0.0001$; see Materials and Methods). 

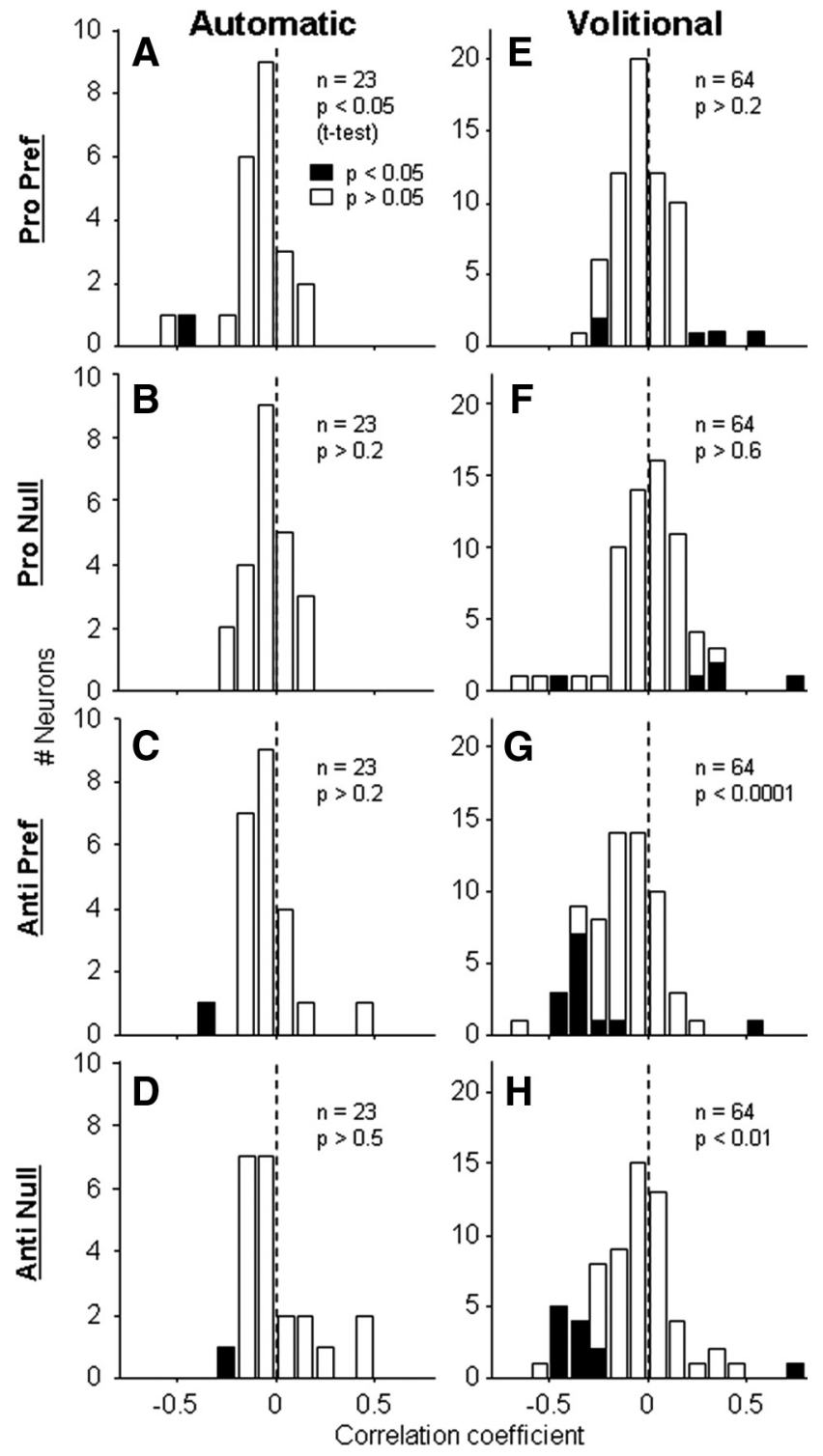

Figure 8. Correlation between reaction times and caudate prestimulus activity (overlap condition). A-D, Automatic neurons. $\boldsymbol{E}-\boldsymbol{H}$, Volitional neurons. $\boldsymbol{A}, \boldsymbol{E}$, Prosaccades (Pro) toward the preferred (Pref) direction. $\boldsymbol{B}, \boldsymbol{F}$, Prosaccades toward the null direction. $\boldsymbol{C}, \boldsymbol{G}$, Antisaccades (Anti) toward the preferred direction. $\boldsymbol{D}, \boldsymbol{H}$, Antisaccades toward the null direction. Positive and negative values of correlation coefficients indicate lower and higher prestimulus activity on trials with shorter reaction times, respectively. Black bars indicate neurons with significant correlation (Pearson's partial correlation coefficient, $p<0.05$; see Materials and Methods for details).

Second, we compared the prestimulus activity of saccaderelated caudate neurons between trials when monkeys generated correct antisaccades and trials when they generated direction error saccades in response to the same stimulus. The majority of neurons in this analysis were derived from one monkey (monkey O) whose direction error rates were sufficient to collect enough direction error trials (see Fig. 9 legend for the numbers of neurons from monkey $\mathrm{O}$ ). We performed multiple linear regression analyses with the main factors of behavioral responses (correct/error) and fixation durations. For automatic neurons, the distributions of regression coefficients for behavioral responses were centered at zero when antisaccades were required toward their preferred $(t$ test: $\mathrm{df}=10, t=-1.7, p>0.1)($ Fig. $9 A)$ or null $(\mathrm{df}=7, t=$ $-0.64, p>0.5$ ) (Fig. 9B) direction, indicating that their pre-

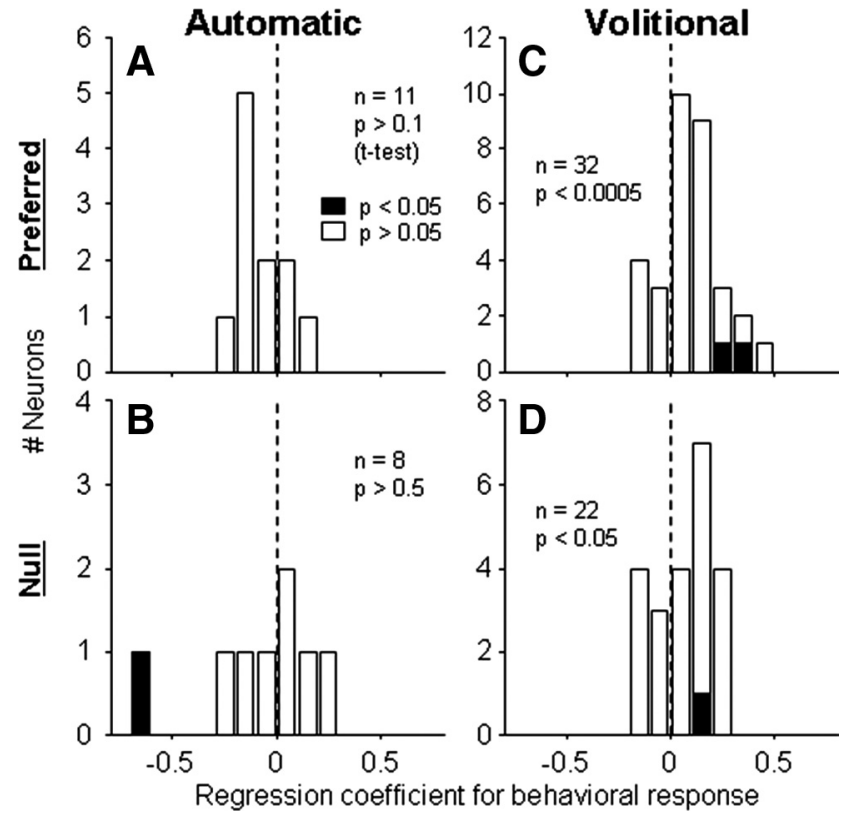

Figure 9. Antisaccade performance dependence of caudate prestimulus activity (overlap condition). $\boldsymbol{A}, \boldsymbol{B}$, Regression coefficients for behavioral response (correct/direction error) in automatic neurons when antisaccades were required toward their preferred $(\boldsymbol{A})$ and null $(\boldsymbol{B})$ direction. $\boldsymbol{C}, \boldsymbol{D}$, Volitional neurons on trials requiring antisaccades toward their preferred $(\boldsymbol{C})$ and null ( $\boldsymbol{D}$ ) direction. Positive and negative values of regression coefficients for behavioral response indicate higher and lower prestimulus activity on correct trials compared with direction error trials, respectively. The numbers of neurons from monkey 0 are as follows: $10(\boldsymbol{A}), 7(\boldsymbol{B}), 28(\boldsymbol{C})$, 21 (D). Black bars indicate neurons with regression coefficients whose $95 \%$ confidence intervals did not include zero.

stimulus activity was insensitive to behavioral outcome. On the other hand, for volitional neurons, the distributions of regression coefficients for behavioral responses were biased toward positive values when antisaccades were required toward both preferred ( $\mathrm{df}=31, t=4.0, p<0.0005$ ) (Fig. $9 \mathrm{C}$ ) and null ( $\mathrm{df}=21, t=2.6$, $p<0.05$ ) (Fig. 9D) directions at the population level, although analyses in most individual neurons did not reach statistical significance presumably because of the small number of direction error trials. This indicates that the population of volitional neurons had stronger prestimulus activity on correct antisaccade trials compared with direction error trials. The regression coefficients for behavioral responses were not different between trials with required antisaccades toward the preferred and null directions for a subset of volitional neurons with enough direction error trials for both directions (paired $t$ test: $\mathrm{df}=18, t=0.51, p>$ $0.6)$. The enhanced activity of volitional neurons on correct antisaccade trials became spatially specific before saccade initiation (average \pm SD of regression coefficients for behavioral responses calculated during the presaccade period; preferred direction: $0.39 \pm 0.21$, null direction: $0.03 \pm 0.24$, paired $t$ test: $\mathrm{df}=18, t=$ $6.49, p<0.0001)$. We confirmed these results for volitional neurons with contralateral saccade direction preferences but failed to detect the enhanced prestimulus activity on correct antisaccade trials compared with direction error trials in volitional neurons with ipsilateral saccade direction preferences (supplemental Fig. 6, available at www.jneurosci.org as supplemental material).

The selective activation of volitional neurons on correct antisaccade trials is supported further by the following analysis. On trials with required antisaccades toward the preferred direction, regression coefficients for behavioral responses were correlated with saccade direction indices on antisaccade trials when we an- 
alyzed automatic and volitional neurons together (Pearson's $r=0.45, p<0.005$, $n=43$ ), indicating that the prestimulus activity was more sensitive to behavioral responses for neurons facilitating correct volitional saccade commands more strongly. This relationship was very similar to what we found between regression coefficients for task instruction and antisaccade direction indices (see above, Selective activation of volitional neurons by antisaccade instruction). Although we found a similar tendency on trials with required antisaccades toward the null direction, the analysis did not reach statistical significance $(r=0.35, p>0.05, n=30)$.

The bidirectional correlation in volitional neurons is consistent with our hypothesis that nonspatial signals, such as task instruction and elapsed time from fixation initiation, influence the prestimulus activity of volitional neurons, which in turn affects preparatory set for upcoming antisaccade initiation.

\section{Discussion}

We demonstrated that saccade-related neurons in the caudate nucleus had prestimulus activity regulated by several factors influencing behavioral outcome. For volitional neurons, their prestimulus activity was enhanced by an antisaccade instruction (Fig. 6), elapsed time from fixation initiation, and a fixation gap (Fig. 7). Furthermore, their enhanced prestimulus activity was correlated with antisaccade behavior (Figs. 8, 9). For automatic neurons, their prestimulus activity was also enhanced by elapsed time from fixation initiation and a fixation gap (Fig. 7). However, their prestimulus activity was not facilitated by an antisaccade instruction (Fig. 6), nor did it correlate with antisaccade behavior (Figs. 8, 9). We suggest that the prestimulus activity of caudate neurons presets BG circuits in favor of volitional actions to compete against automatic actions even before automatic and volitional commands are programmed with spatial information.

\section{Creation of caudate preparatory signals} for volitional actions

The prestimulus activity of volitional neurons was facilitated by an antisaccade instruction (Fig. 6). This is partially consistent with a previous report in which caudate neurons were recorded during the antisaccade paradigm but not dissociated based on automatic/volitional saccade encodings (antisaccade instruction preferences for contralateral, but not ipsilateral, saccade-preferred neurons) (Ford and Everling, 2009). However, despite the

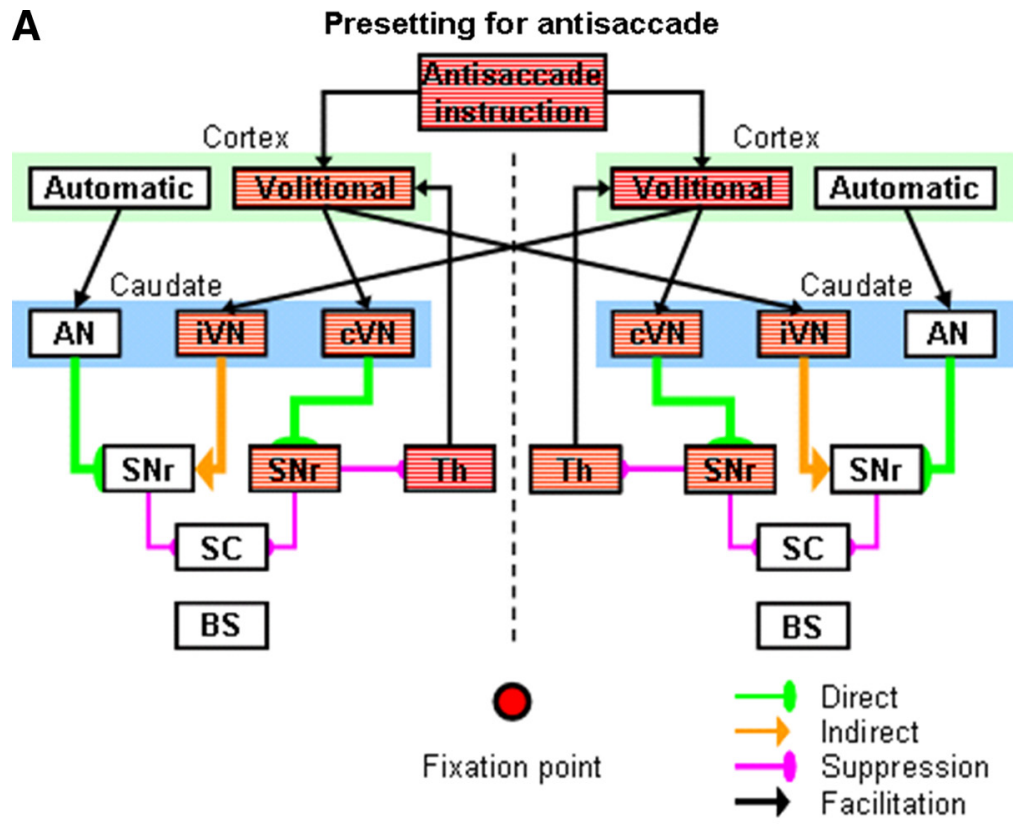

B Antisaccade initiation

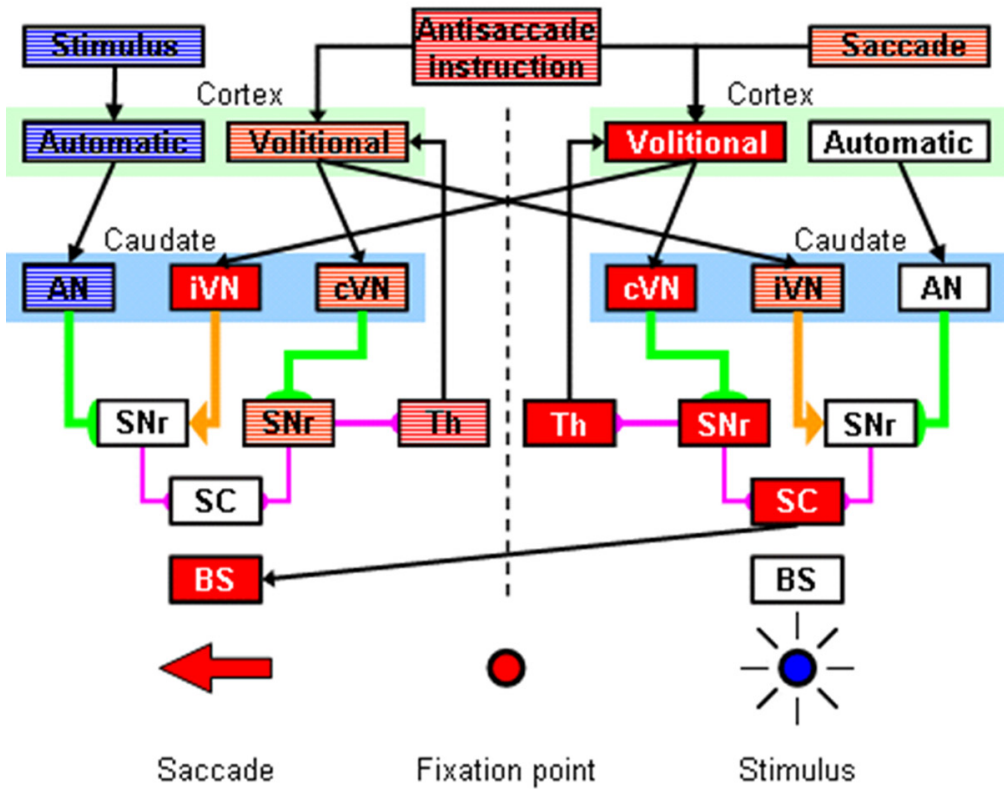

Figure 10. Hypothetical schematic diagram for the role of caudate preparatory activity in conflict resolution between automatic and volitional saccades. $A$, Before stimulus appearance, an antisaccade instruction given by fixation point color activates the cortex-basal ganglia circuits that facilitate volitional saccades in both hemispheres. These circuits consist of cortical volitional neurons (Volitional), caudate volitional neurons with contralateral saccade preferences (cVN), a subset of substantia nigra pars reticulata neurons ( $\mathrm{SNr}$ ), and thalamic neurons (Th). cVNs give rise to the direct pathway so that the cortical-BG circuits form positive feedback loops and develop preparatory signals for antisaccades. Cortical volitional neurons also project to caudate volitional neurons with ipsilateral saccade preferences (iVN) in the opposite hemisphere. iVNs give rise to the indirect pathway. Caudate automatic neurons (AN) receive cortical automatic neurons (Automatic) and give rise to the direct pathway. Inappropriate automatic preparatory signals carried by the automatic pathway are attenuated by signals issued by iVNs in $\mathrm{SNr}$ neurons receiving input from both ANs and iVNs. $\boldsymbol{B}$, After stimulus appearance, erroneous automatic saccade commands driven by stimulus appearance (Stimulus) reach to the BG earlier than correct volitional saccade commands in the opposite hemisphere (Saccade). However, the automatic saccade commands are attenuated by the preparatory suppression signal issued by iVNs, even before iVNs receive volitional saccade commands. After cortical volitional neurons receive correct antisaccade commands, they activate cVNs in the same hemisphere and iVNs in the opposite hemisphere. iVNs attenuate erroneous automatic saccade commands further, while cVNs enhance volitional saccade commands through the cortex-BG loop to release the superior colliculus (SC) for correct antisaccade initiation before an erroneous automatic saccade is triggered. BS, Brainstem. 
fact that the information of task instructions was available at fixation point appearance (Fig. $1 B$ ), the antisaccade instruction preferences of volitional neurons only emerged gradually before stimulus appearance (Fig. $6 C, D$ ). Because fixation point color must be represented in the visual cortex immediately after fixation initiation, it is reasonable to speculate that signals encoding fixation point color or task instruction fed into the caudate nucleus well before the output of volitional neurons started discriminating between prosaccade and antisaccade instructions. It has been shown that caudate neurons show bistable membrane potentials (up/down states), and to change membrane potentials from down to up state they need to integrate excitatory input from a large number of cortical neurons (Wilson, 2008). Accordingly, we speculate that cortical input carrying fixation point color or task instruction alone was not strong enough to activate volitional neurons immediately following fixation initiation on antisaccade trials (Fig. 6C). Instead, we suggest that volitional neurons manifested their antisaccade instruction preferences by integrating signals encoding fixation point color or task instructions (Fig. 6B) and those encoding elapsed time from fixation initiation (Fig. 7D).

\section{Conflict resolution between automatic and volitional actions}

The selective activation of volitional neurons on antisaccade trials is presumably advantageous for correct antisaccade performance. However, it is still unclear how caudate preparatory signals contribute to resolving the conflict between automatic and volitional saccade commands imposed by the antisaccade paradigm. Here, we propose a simple hypothetical model (Fig. 10) by taking into account anatomical and physiological evidence to promote future research. In the following discussion, we consider volitional neurons preferring contralateral and ipsilateral saccades separately, although their firing characteristics were similar (supplemental Figs. 2-6, available at www.jneurosci.org as supplemental material). We ignore automatic neurons preferring ipsilateral saccades here because they were rare in our dataset (Table 1).

We have hypothesized previously (Watanabe and Munoz, 2009) that automatic neurons (ANs) and contralateral saccadepreferred volitional neurons (cVNs) give rise to the direct pathway (represented by green arrows in Fig. 10) that facilitates saccade initiation by monosynaptic connections with the $\mathrm{SNr}$, the output structure of the oculomotor BG, while ipsilateral saccade-preferred volitional neurons (iVNs) give rise to the indirect pathway that suppresses saccade initiation by polysynaptic connections with the $\mathrm{SNr}$ (represented by orange arrows in Fig. 10) (Hikosaka et al., 2000). Signals issued by iVNs compete against those issued by ANs in the $\mathrm{SNr}$. $\mathrm{SNr}$ neurons receiving input from ANs send feedforward projections to the SC, while those receiving input from $\mathrm{cVNs}$ send feedforward projections to the SC and feedback projections to the cortex via thalamus.

Note that the two asymmetric components between pathways controlling automatic and volitional saccades (the indirect pathway originating from iVNs and the feedback projection through the thalamus) are not based on anatomical evidence but rather are designed to be as simple as possible to capture recent physiological findings. The suppression of the automatic pathway by iVNs is consistent with the following reports. Neurons in BG nuclei composing the indirect pathway have strongly modulated activity when automatic saccade suppression is required (Isoda and Hikosaka, 2008; Yoshida and Tanaka, 2009). Furthermore, saccade suppression by caudate microstimulation is stronger for prosaccades than for antisaccades (Watanabe and Munoz, 2010).
The feedback connection for the volitional pathway to the cortex via thalamus is based on a recent report; neurons in parts of the thalamus (ventrolateral and ventroanterior nuclei) are involved in antisaccades more strongly than prosaccades (Kunimatsu and Tanaka, 2010). The separate control systems for automatic and volitional actions are consistent with the following behavioral phenomenon; when sensory-driven actions are required suddenly while subjects are preparing internally driven actions, the sensory-driven actions cannot take advantage of preexisting preparation for the internally driven actions (Obhi and Haggard, 2004).

The preparatory activity of caudate neurons could contribute to conflict resolution between automatic and volitional saccades even before actual saccade directions are defined by stimulus appearance if the asymmetric structure of the cortex-BG circuits is implemented. After an antisaccade instruction is given by fixation point color, cVNs and iVNs increase activity with time in both hemispheres (Fig. 10A). The feedback loop connecting cVNs and cortical volitional neurons (Fig. 10, "Volitional") enhances the preparatory activity of cVNs and iVNs further. The enhanced signals issued by iVNs attenuate inappropriate preparatory signals issued by ANs in the $\mathrm{SNr}$. This suppression effect is also important to attenuate erroneous automatic saccade commands that reach the BG from cortical automatic neurons (Fig. 10, "Automatic") earlier than correct volitional saccade commands after stimulus appearance (Fig. $10 \mathrm{~B}$ ). To compensate the temporal disadvantage of volitional saccade commands, the cortex-BG loop facilitates volitional saccade commands further to initiate correct antisaccades before erroneous automatic saccades are triggered.

To account for other physiological findings, additional elements must be required in the model. Partial connections from cortical volitional neurons to caudate automatic neurons might be necessary to account for the weak postsaccadic activity of caudate automatic neurons (Fig. 5B). Projections from the SNr to the SC and/or thalamus in the opposite hemisphere (Jiang et al., 2003; Cebrian et al., 2005) might be required to account for ipsilateral saccade suppression by caudate microstimulation (Watanabe and Munoz, 2010). The pathway controlling automatic saccades might also form a feedback loop via thalamus, although such circuit might not be critical for saccade initiation (Sommer and Wurtz, 2002; Tanaka, 2006; Kunimatsu and Tanaka, 2010).

\section{Preparatory signals outside BG}

Preparatory activity during the prosaccade and antisaccade paradigm has been reported in a number of structures outside the BG. A subset of neurons in the prefrontal cortex (Everling and Desouza, 2005) and supplemental eye field (Schlag-Rey et al., 1997) have enhanced preparatory activity on antisaccade trials compared with prosaccade trials. Because of the similarity between these cortical neurons and caudate volitional neurons, they might form neural circuits that correspond to the cortex-BG loops for volitional saccades in the model (Fig. 10). In contrast, neurons in the lateral intraparietal area that presumably issue automatic saccade commands do not have instruction preferences in their preparatory activity (Gottlieb and Goldberg, 1999). The activity of caudate automatic neurons might reflect this cortical activity.

There are also neurons whose preparatory activity is weaker on antisaccade trials compared with prosaccade trials in the prefrontal cortex (Everling and Desouza, 2005), frontal eye field (FEF) (Everling and Munoz, 2000), and SC (Everling et al., 1999). Because our model cannot explain this attenuated preparatory 
activity on antisaccade trials, additional elements must be required in the model. It has been hypothesized that the attenuated preparatory activity in the FEF and SC can prevent phasic visual activity triggered by stimulus appearance from initiating erroneous saccades toward the stimulus (Everling et al., 1999; Everling and Munoz, 2000). This attenuation might be mediated by a subset of neurons in the same structures controlling fixation (Hanes et al., 1998; Everling et al., 1999; Munoz and Everling, 2004). It is also possible that the subthalamic nucleus (STN) that suppresses the FEF and SC via SNr is involved in this attenuation, because a subset of STN neurons has fixation-related activity (Matsumura et al., 1992). It has also been suggested that a pathway that links the cerebral cortex and SNr via STN (hyperdirect) (Nambu et al., 2002) is critical for conflict resolution between multiple potential responses (Frank et al., 2007).

The discussion described so far is devoted specifically for integrating physiological findings from multiple structures in the effort to understand how the whole oculomotor system establishes preparatory set to perform the antisaccade paradigm. In future research, it will be important to develop this discussion further to address the causes of deficits in volitional action preparation in BG disorders, such as Parkinson's and Huntington's diseases (Cunnington et al., 1997; Johnson et al., 2001), which might account for their antisaccade deficits (Chan et al., 2005; Peltsch et al., 2008; Cameron et al., 2010).

\section{References}

Alexander GE, DeLong MR, Strick PL (1986) Parallel organization of functionally segregated circuits linking basal ganglia and cortex. Annu Rev Neurosci 9:357-381.

Aosaki T, Kimura M, Graybiel AM (1995) Temporal and spatial characteristics of tonically active neurons of the primate's striatum. J Neurophysiol 73:1234-1252.

Apicella P (2007) Leading tonically active neurons of the striatum from reward detection to context recognition. Trends Neurosci 30:299-306.

Apicella P, Scarnati E, Ljungberg T, Schultz W (1992) Neuronal activity in monkey striatum related to the expectation of predictable environmental events. J Neurophysiol 68:945-960.

Bell AH, Everling S, Munoz DP (2000) Influence of stimulus eccentricity and direction on characteristics of pro- and antisaccades in non-human primates. J Neurophysiol 84:2595-2604.

Cameron IG, Watanabe M, Pari G, Munoz DP (2010) Executive impairment in Parkinson's disease: response automaticity and task switching. Neuropsychologia 48:1948-1957.

Cebrian C, Parent A, Prensa L (2005) Patterns of axonal branching of neurons of the substantia nigra pars reticulata and pars lateralis in the rat. J Comp Neurol 492:349-369.

Chan F, Armstrong IT, Pari G, Riopelle RJ, Munoz DP (2005) Deficits in saccadic eye-movement control in Parkinson's disease. Neuropsychologia 43:784-796.

Colebatch JG (2007) Bereitschaftspotential and movement-related potentials: origin, significance, and application in disorders of human movement. Mov Disord 22:601-610.

Connolly JD, Goodale MA, Menon RS, Munoz DP (2002) Human fMRI evidence for the neural correlates of preparatory set. Nat Neurosci 5:1345-1352.

Crist CF, Yamasaki DS, Komatsu H, Wurtz RH (1988) A grid system and a microsyringe for single cell recording. J Neurosci Methods 26:117-122.

Cunnington R, Iansek R, Johnson KA, Bradshaw JL (1997) Movementrelated potentials in Parkinson's disease. motor imagery and movement preparation. Brain 120:1339-1353.

Dafoe JM, Armstrong IT, Munoz DP (2007) The influence of stimulus direction and eccentricity on pro- and anti-saccades in humans. Exp Brain Res 179:563-570.

Dean AF (1981) The variability of discharge of simple cells in the cat striate cortex. Exp Brain Res 44:437-440.

DeAngelis GC, Uka T (2003) Coding of horizontal disparity and velocity by MT neurons in the alert macaque. J Neurophysiol 89:1094-1111.
Dias EC, Bruce CJ (1994) Physiological correlate of fixation disengagement in the primate's frontal eye field. J Neurophysiol 72:2532-2537.

Dorris MC, Munoz DP (1995) A neural correlate for the gap effect on saccadic reaction times in monkey. J Neurophysiol 73:2558-2562.

Everling S, Desouza JF (2005) Rule-dependent activity for prosaccades and antisaccades in the primate prefrontal cortex. J Cogn Neurosci 17:1483-1496.

Everling S, Fischer B (1998) The antisaccade: a review of basic research and clinical studies. Neuropsychologia 36:885-899.

Everling S, Munoz DP (2000) Neuronal correlates for preparatory set associated with pro-saccades and anti-saccades in the primate frontal eye field. J Neurosci 20:387-400.

Everling S, Pare M, Dorris MC, Munoz DP (1998) Comparison of the discharge characteristics of brain stem omnipause neurons and superior colliculus fixation neurons in monkey: implications for control of fixation and saccade behavior. J Neurophysiol 79:511-528.

Everling S, Dorris MC, Klein RM, Munoz DP (1999) Role of primate superior colliculus in preparation and execution of anti-saccades and prosaccades. J Neurosci 19:2740-2754.

Fischer B, Weber H (1992) Characteristics of "anti" saccades in man. Exp Brain Res 89:415-424.

Ford KA, Everling S (2009) Neural activity in primate caudate nucleus associated with pro- and antisaccades. J Neurophysiol 102:2334-2341.

Francois C, Yelnik J, Percheron G (1996) A stereotaxic atlas of the basal ganglia in macaques. Brain Res Bull 41:151-158.

Frank MJ, Samanta J, Moustafa AA, Sherman SJ (2007) Hold your horses: impulsivity, deep brain stimulation, and medication in parkinsonism. Science 318:1309-1312.

Fuchs AF, Robinson DA (1966) A method for measuring horizontal and vertical eye movement chronically in the monkey. J Appl Physiol 21:1068-1070.

Gottlieb J, Goldberg ME (1999) Activity of neurons in the lateral intraparietal area of the monkey during an antisaccade task. Nat Neurosci 2:906-912.

Haggard P (2008) Human volition: towards a neuroscience of will. Nat Rev Neurosci 9:934-946.

Hallett PE (1978) Primary and secondary saccades to goals defined by instructions. Vision Res 18:1279-1296.

Hanes DP, Patterson WF 2nd, Schall JD (1998) Role of frontal eye fields in countermanding saccades: visual, movement, and fixation activity. J Neurophysiol 79:817-834.

Hays AV, Richmond BJ, Optican LM (1982) A UNIX-based multiple process system for real-time data acquisition and control. WESCON Conf Proc 2:1-10.

Hikosaka O, Wurtz RH (1983) Visual and oculomotor functions of monkey substantia nigra pars reticulata. IV. Relation of substantia nigra to superior colliculus. J Neurophysiol 49:1285-1301.

Hikosaka O, Sakamoto M, Usui S (1989a) Functional properties of monkey caudate neurons. I. activities related to saccadic eye movements. J Neurophysiol 61:780-798.

Hikosaka O, Sakamoto M, Usui S (1989b) Functional properties of monkey caudate neurons. III. activities related to expectation of target and reward. J Neurophysiol 61:814-832.

Hikosaka O, Sakamoto M, Miyashita N (1993) Effects of caudate nucleus stimulation on substantia nigra cell activity in monkey. Exp Brain Res 95:457-472.

Hikosaka O, Takikawa Y, Kawagoe R (2000) Role of the basal ganglia in the control of purposive saccadic eye movements. Physiol Rev 80:953-978.

Isoda M, Hikosaka O (2008) Role for subthalamic nucleus neurons in switching from automatic to controlled eye movement. J Neurosci 28:7209-7218.

Itoh H, Nakahara H, Hikosaka O, Kawagoe R, Takikawa Y, Aihara K (2003) Correlation of primate caudate neural activity and saccade parameters in reward-oriented behavior. J Neurophysiol 89:1774-1783.

Jiang H, Stein BE, McHaffie JG (2003) Opposing basal ganglia processes shape midbrain visuomotor activity bilaterally. Nature 423:982-986.

Johnson KA, Cunnington R, Iansek R, Bradshaw JL, Georgiou N, Chiu E (2001) Movement-related potentials in huntington's disease: movement preparation and execution. Exp Brain Res 138:492-499.

Judge SJ, Richmond BJ, Chu FC (1980) Implantation of magnetic search coils for measurement of eye position: an improved method. Vision Res 20:535-538. 
Kimura M, Rajkowski J, Evarts E (1984) Tonically discharging putamen neurons exhibit set-dependent responses. Proc Natl Acad Sci U S A 81:4998-5001.

Kimura M, Aosaki T, Hu Y, Ishida A, Watanabe K (1992) Activity of primate putamen neurons is selective to the mode of voluntary movement: visually guided, self-initiated or memory-guided. Exp Brain Res 89:473-477.

Kunimatsu J, Tanaka M (2010) Roles of the primate motor thalamus in the generation of antisaccades. J Neurosci 30:5108-5117.

Lee IH, Assad JA (2003) Putaminal activity for simple reactions or selftimed movements. J Neurophysiol 89:2528-2537.

Marino RA, Rodgers CK, Levy R, Munoz DP (2008) Spatial relationships of visuomotor transformations in the superior colliculus map. J Neurophysiol 100:2564-2576.

Matsumura M, Kojima J, Gardiner TW, Hikosaka O (1992) Visual and oculomotor functions of monkey subthalamic nucleus. J Neurophysiol 67:1615-1632.

Mikula S, Trotts I, Stone JM, Jones EG (2007) Internet-enabled highresolution brain mapping and virtual microscopy. Neuroimage 35:9-15.

Miyashita N, Hikosaka O (1996) Minimal synaptic delay in the saccadic output pathway of the superior colliculus studied in awake monkey. Exp Brain Res 112:187-196.

Munoz DP, Everling S (2004) Look away: the anti-saccade task and the voluntary control of eye movement. Nat Rev Neurosci 5:218-228.

Munoz DP, Waitzman DM, Wurtz RH (1996) Activity of neurons in monkey superior colliculus during interrupted saccades. J Neurophysiol 75:2562-2580.

Nambu A, Tokuno H, Takada M (2002) Functional significance of the cortico-subthalamo-pallidal 'hyperdirect' pathway. Neurosci Res 43:111117.

Obhi SS, Haggard P (2004) Internally generated and externally triggered actions are physically distinct and independently controlled. Exp Brain Res 156:518-523.

Oswal A, Ogden M, Carpenter RH (2007) The time course of stimulus expectation in a saccadic decision task. J Neurophysiol 97:2722-2730.

Pare M, Munoz DP (1996) Saccadic reaction time in the monkey: advanced preparation of oculomotor programs is primarily responsible for express saccade occurrence. J Neurophysiol 76:3666-3681.

Peltsch A, Hoffman A, Armstrong I, Pari G, Munoz DP (2008) Saccadic impairments in Huntington's disease. Exp Brain Res 186:457-469.

Prince SJ, Pointon AD, Cumming BG, Parker AJ (2002) Quantitative analysis of the responses of $\mathrm{V} 1$ neurons to horizontal disparity in dynamic random-dot stereograms. J Neurophysiol 87:191-208.

Robinson DA (1963) A method of measuring eye movement using a scleral search coil in a magnetic field. IEEE Trans Biomed Eng 10:137-145.
Romo R, Scarnati E, Schultz W (1992) Role of primate basal ganglia and frontal cortex in the internal generation of movements. II. Movementrelated activity in the anterior striatum. Exp Brain Res 91:385-395.

Saslow MG (1967) Effects of components of displacement-step stimuli upon latency for saccadic eye movement. J Opt Soc Am 57:1024-1029.

Schlag-Rey M, Amador N, Sanchez H, Schlag J (1997) Antisaccade performance predicted by neuronal activity in the supplementary eye field. Nature 390:398-401.

Schmolesky MT, Wang Y, Hanes DP, Thompson KG, Leutgeb S, Schall JD, Leventhal AG (1998) Signal timing across the macaque visual system. J Neurophysiol 79:3272-3278.

Schultz W, Romo R (1992) Role of primate basal ganglia and frontal cortex in the internal generation of movements. I. Preparatory activity in the anterior striatum. Exp Brain Res 91:363-384.

Schultz W, Apicella P, Scarnati E, Ljungberg T (1992) Neuronal activity in monkey ventral striatum related to the expectation of reward. J Neurosci 12:4595-4610.

Sommer MA, Wurtz RH (2002) A pathway in primate brain for internal monitoring of movements. Science 296:1480-1482.

Tachibana Y, Kita H, Chiken S, Takada M, Nambu A (2008) Motor cortical control of internal pallidal activity through glutamatergic and GABAergic inputs in awake monkeys. Eur J Neurosci 27:238-253.

Takikawa Y, Kawagoe R, Hikosaka O (2002) Reward-dependent spatial selectivity of anticipatory activity in monkey caudate neurons. J Neurophysiol 87:508-515.

Tanaka M (2006) Inactivation of the central thalamus delays self-timed saccades. Nat Neurosci 9:20-22.

Tolhurst DJ, Movshon JA, Thompson ID (1981) The dependence of response amplitude and variance of cat visual cortical neurones on stimulus contrast. Exp Brain Res 41:414-419.

Watanabe M, Munoz DP (2009) Neural correlates of conflict resolution between automatic and volitional actions by basal ganglia. Eur J Neurosci 30:2165-2176.

Watanabe M, Munoz DP (2010) Saccade suppression by electrical microstimulation in monkey caudate nucleus. J Neurosci 30:2700-2709.

Wilson CJ (2008) Up and down states. Scholarpedia 3:1410.

Wilson CJ, Chang HT, Kitai ST (1990) Firing patterns and synaptic potentials of identified giant aspiny interneurons in the rat neostriatum. J Neurosci 10:508-519.

Yoshida A, Tanaka M (2009) Enhanced modulation of neuronal activity during antisaccades in the primate globus pallidus. Cereb Cortex 19:206217. 